\title{
EVALUATION OF EFFICIENCY OF VARIOUS MATERIALS TO SHIELD FROM RADIATION IN SPACE USING THE MONTE CARLO TRANSPORT CODE CALLED FLUKA
}

\author{
A Thesis \\ presented to \\ the Faculty of California Polytechnic State University, \\ San Luis Obispo
}

In Partial Fulfillment

of the Requirements for the Degree

Master of Science in Aerospace Engineering

by

Roman Savinov

December 2016 
(C) 2016

Roman Savinov

ALL RIGHTS RESERVED 


\section{COMMITTEE MEMBERSHIP}

TITLE:

AUTHOR:

DATE SUBMITTED:

COMMITTEE CHAIR:

COMMTTEE MEMBER:

Eric Mehiel, Ph.D.

Professor of Aerospace Engineering

COMMITTEE MEMBER: Jodi Christiansen, Ph.D.

Associate Professor of Physics

COMMITTEE MEMBER: Jennifer Klay, Ph.D. Called FLUKA

Roman Savinov

November 2016

Kira Abercromby, Ph.D.
Associate Professor of Physics

Evaluation of Efficiency of Various Materials to Shield from

Radiation in Space Using the Monte Carlo Transport Code

Associate Professor of Aerospace Engineering 


\begin{abstract}
Evaluation of Efficiency of Various Materials to Shield from Radiation in Space Using the Monte Carlo Transport Code Called FLUKA

Roman Savinov
\end{abstract}

The purpose of this study is to improve spacecraft shielding from radiation in space. It focuses on the evaluation of shielding efficiency of different materials. The efficiency of a shield is evaluated by the dose profile within the shield and the amount of dose absorbed by a target using the Monte Carlo transport code called FLUKA. The output of this code is validated by recreating the experiments from published papers and comparing the results. Once the FLUKA's output is validated, the efficiency of sixteen materials, subject to SPE and GCR sources, are evaluated. The efficiency comparison is made by fixing the area density of a shield.

It was found that polyethylene, water, carbon and silicon outperform aluminum - the primary metal used in spacecraft. In case of composite shield, made of layers of different materials, the 3Carb-9Al combination has better performance than the shield made just of aluminum. This holds true for both Solar Particle Events (SPEs) and Galactic Cosmic Ray (GCR). However, the choice of material is more efficient at shielding from SPE particles rather than from GCR. In case of GCR, the choice of materials is found to have rather small effect on the efficiency of a shield. The percent difference between the rate of dose absorption by a target, shielded by different materials, is within about $9 \%$. Secondary particles make a significant contribution to the target's dose. For SPEs, the secondary particles are primarily electrons and neutrons. For GCRs, the secondary particles are primarily pions, $\alpha$-particles and electrons. Protons contribute more than $50 \%$ to the target's dose in both cases. 


\section{ACKNOWLEDGMENTS}

I would like to thank Dr. Kira Abercromby for her support, guidance and encouragement through my preparation of this work. I greatly appreciate her endless patience with my slow advance and her continual guidance that helped me stay on track all this time.

Also, I would like to thank Dr. Jodi Christiansen for teaching me the basics of particle physics and for clarifying some physical concepts important to this work.

Lastly, I would like to thank Dr. Jennifer Klay and Dr. Eric Mehiel for agreeing to be in my committee. I appreciate their patience with me. 


LIST OF TABLES ... Page

7.1 FLUKA Validation By Repeating The Aghara Paper Experiment...................................................20

7.1.1 The Source

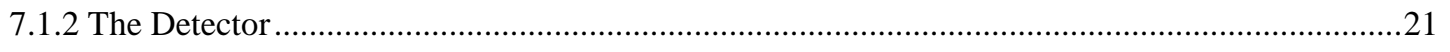

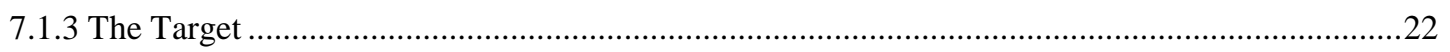

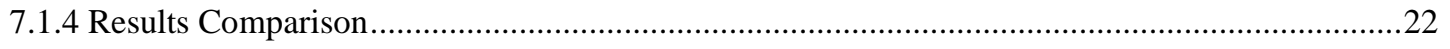

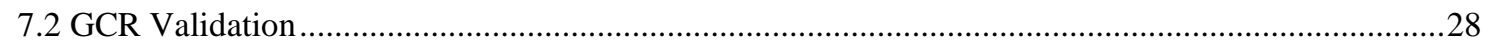




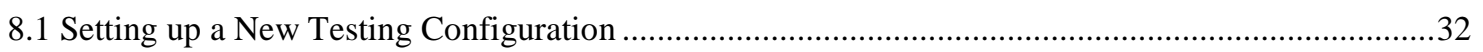

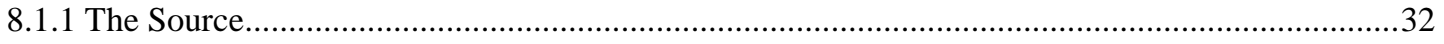

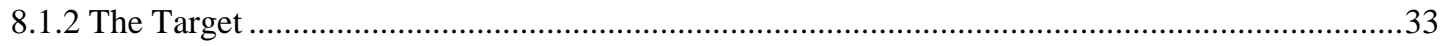

8.2 Effect of the Distance Between Souce and Target on the Resultant Absorbed Dose .........................36

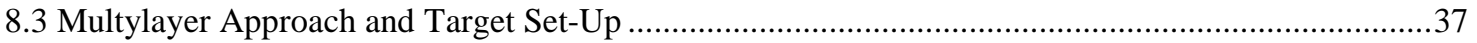

8.4 Choosing the Solar Event to Use as a Source .......................................................................40

8.5 Dose Profiles Within Diferent Materials and Resultant Target Doses ..........................................42

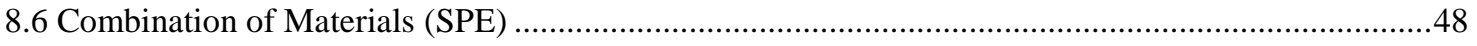

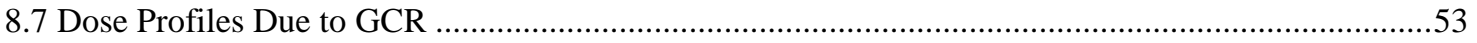

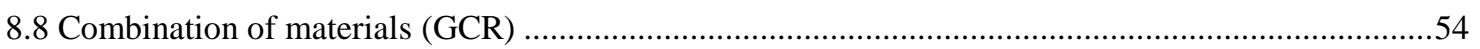

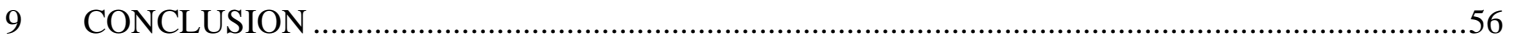

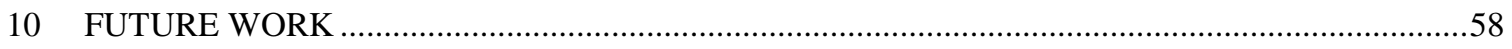

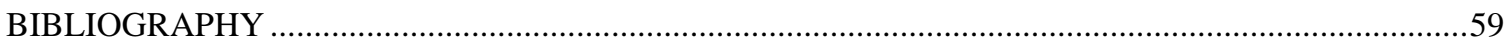




\section{LIST OF TABLES}

Table Page

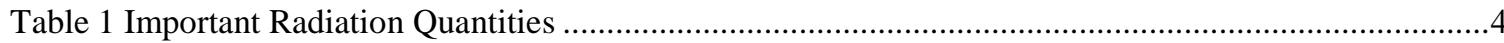

Table 2 Difference Between Uni- and Omnidirectional flux a fluences .......................................................5

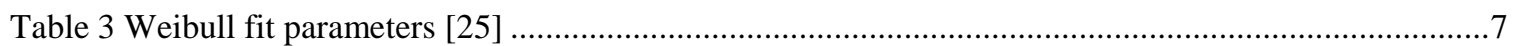

Table 4 Band Fit Parameters for Some SPEs Used in This Study [22] ......................................................

Table 5 Example career Effective dose limits for 1-year missions for a 3\% REID [7]................................10

Table 6 Radiation Limits for Other Space Agencies [7] .......................................................................10

Table 7 Spectral Weibull parameters for two SPE events [2] ......................................................................21

Table 8 MSD Values* for Fluxes Obtained by FLUKA, with eq.15 and from Aghara’s Paper ..................25

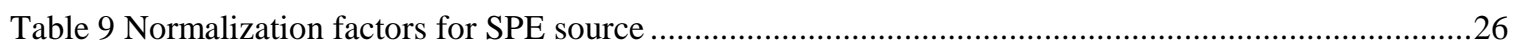

Table 10 MSD Values for Doses in figure 11 to compare FLUKA, MCNPX and OLTARIS results ..........28

Table 11 ICRU Elemental composition (wt.\%) of soft tissues in the human body used in FLUKA

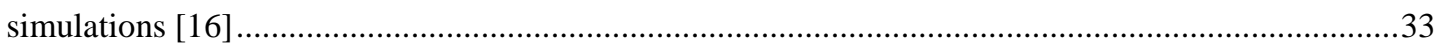

Table 12 Inelastic Scattering Length Into ICRU Tissue as a Function of Ion and Energy [16] ....................34

Table 13 Comparison between fluences obtained by FLUKA, Rojdev and Band .......................................39

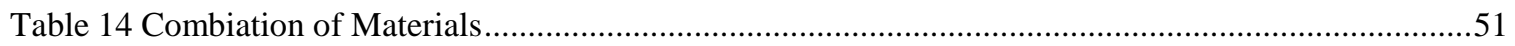




\section{LIST OF FIGURES}

Figure

Page

Figure 1 Differential spectrum of GCR at solar min and max (MeV/in is MeV per incident particle [14].....1

Figure 2 Large SPEs (proton fluence $>108 \mathrm{~cm}-2$ at E>30, $60 \& 100 \mathrm{MeV}$ ) as a function of time [13]........2

Figure 3 SPE integral spectra of larger solar particle events from 1956 to 1989 [1]...............................3

Figure 4 The Z/(A $\rho)$ Ratio Values for Different Materials (Hydrogen is in liquid form) ...........................11

Figure 5 Different ions tracks in nuclear emulsion. Note the increase in number of $\delta$-rays along the track with increasing atomic number [6] ................................................................. 12

Figure 6 Dependence of the quality factor on a particle’s energy for several GCR nuclei [6]....................13

Figure 7 (a) Constructive Solid Geometry, (b) example of a boolean geometry tree. Courtesy of

N. Stewart.

Figure 8 Target from the Aghara paper. (a) 3D model, (b) how it is modeled in FLUKA.

Figure 9 Fit of the FLUKA-generated Fluences with Fluences from the Aghara's Paper and

Theoretical Fluences

Figure 10 Percent uncertainty in the USRBDX result

Figure 11 Dose distribution within the water slab shielded by $10 \mathrm{~g} / \mathrm{cm}^{2}$ of aluminum shield for:

(a) 56 Webber, (b) 72 LaRC, (c) 89 Weibull and (d) 91 Carrington

Figure 12 (a) FLUKA's GCR source proton spectrum, $\alpha$-particles, carbon and iron ions,

(b) FLUKA's output of the flux scoring detector 29

Figure 13 Fluka model of RAD.

Figure 14 Overlap of the RAD measurements with fluka simulation output

Figure 15 Two Models of a Spacecraft with a "human" target inside. (a) - realistic. (b) - simplified 35

Figure 16 Difference in Dose Absorbed by the Target Between "Realistic" and "Simplified"

Models for Four SPE Events

Figure 17 Influence of distance between source and target on dose absorbed

Figure 18 Validation of Multilayer Approach to Find Dose Within Shield (source: 1956 Webber). 38

Figure 19 Comparison of the Oct 1989 Band Fits: Rojdev, Theoretical and FLUKA 
Figure 20 Comparison in Dose Profile for Aluminum Shield Between FLUKA and Rojdev Paper Results. Comparison Between Aghara and Spherical target Set-Ups (source: 1989 Band Fit)

Figure 21 Proton Fluence of the Largest SPEs (Band Fit)

Figure 22 Dose Profile Within $100 \mathrm{~g} / \mathrm{cm}^{2}$ Thick Aluminum Shield Due to 8 Largest SPEs: (a) Most

Influential, (b) Least Influential.

Figure 23 Dose Absorbed by the Target Due to 9 Largest SPEs. (a) Most Influential,

(b) Least Influential

Figure 24 The Simplified Model to Obtain the Dose Profile Within the Shield

Figure 25 Dose Profiles in Different Materials (SPE source: 1956) ...........................................................43

Figure 26 Dose Profiles in Different Materials (SPE source: 1989)

Figure 27 (a) Fluence of protons, electrons and neutrons between shield and target,

(b) Proton fluence between some shield layers. The source fluence is added for comparison. (Aluminum. Source: 1989) ......

Figure 28 Dose Absorbed by Target for the 1956 and 1989 SPEs (Shield: 100g/cm2).

Total doses are given on top of each bar. Values for protons and electrons are also included. The area on the background is Z/A with own axis on the right . .46

Figure 29 (a) Dose Absorbed by Target. Mass of a Shield is Added Instead of Z/A

(SPE source: 1989. Shield: $100 \mathrm{~g} / \mathrm{cm}^{2}$.

Figure 30 Dose Distribution Within a $100 \mathrm{~g} / \mathrm{cm} 2$ Shield Constructed Out of the Layers of

Different Materials . .50

Figure 31 Target Doses Absorbed with Composite Shield of 100 and $10 \mathrm{~g} / \mathrm{cm}^{2}$ Thick.

(SPE source: 1989)

Figure 32 Dose Absorbed by Target Behind $10 \mathrm{~g} / \mathrm{cm} 2$ of a Shield Made of Single Material

(GCR Source) 54

Figure 33 Target Doses Absorbed with Composite Shield of $10 \mathrm{~g} / \mathrm{cm}^{2}$ Thick. (GCR source). 55 


\section{LIST OF TERMS}
A $\quad-\quad$ Atomic Mass Number
$\mathrm{A}_{\mathrm{r}} \quad-\quad$ Relative Atomic Mass
D _ - $\quad$ Absorbed Dose
GCR - $\quad$ Galactic Cosmic Rays
GLE - - Ground Level Enhancement
CERN - $\quad$ European Organization for Nuclear Research
CMEs - $\quad$ Coronal Mass Ejections
EXP - $\quad$ Exponential in Proton Rigidity
spectral fitting methodology
FDs _ - Forbush Decreases
ICRU - $\quad$ International Commission on Radiation Units
J $\quad-\quad$ Differential Flux
$\mathrm{J}(>\mathrm{E}) \quad-\quad$ Integral Flux
LET _ - Linear Energy Transfer
MSD - $\quad$ Mean Square Deviation
MSL _ - Mars Science Laboratory
Q $\quad-\quad$ Quality Factor
RAD - $\quad$ Radiation Assessment Detector
RBE - $\quad$ Relative Biological Effectiveness
REID - $\quad$ Risk of Exposure Induced Death
SEP $\quad-\quad$ Solar Energetic Particles
SPE - - Solar Particle Event
Z $\quad-\quad$ Atomic Number
$\Phi \quad$ - $\quad$ Fluence
$\sigma \quad-\quad$ Total Nuclear Reaction Cross Section 


\section{INTRODUCTION}

\subsection{Radiation Background}

For space flights beyond the Earth's magnetosphere, both the crew and the spacecraft equipment faces a significant hazard from the natural ionizing radiation environment. The two sources of this radiation are Galactic Cosmic Rays (GCRs) and radiation from the Sun [1]. Both sources consist of energetic protons, $\alpha$ particles and heavy ions.

GCRs originate outside our Solar System and possibly are the result of supernovae explosions. Protons represent the largest component of GCRs (87\%); $\alpha$-particles contribute about 12\%; high-energy nuclei component of GCR (HZE - High Z\# and Energy) contributes about 1\% [4]. HZE are atoms with atomic number (Z) greater than 2 and with stripped off atomic electrons [] $]$. Although the total GCR flux is a very small fraction of HZE, their effect far outweighs their number. This is because the energy deposited by an HZE particle in target is proportional to the square of it's nuclear charge. In addition, the biological effect of an HZE particle is more important than that of a lightweight particle such as proton [1]. Most of the GCR consist of particles with atomic numbers that range between 1 and 28. Their energies range from less than 1 to more than $10^{4} \mathrm{MeV} / \mathrm{amu}$ with a median energy of about $1000 \mathrm{MeV} / \mathrm{amu}$ [6]. Figure 1 shows spectra of four ions present in GCR. Cosmic rays are nearly isotropic meaning they come to the Solar System from every direction

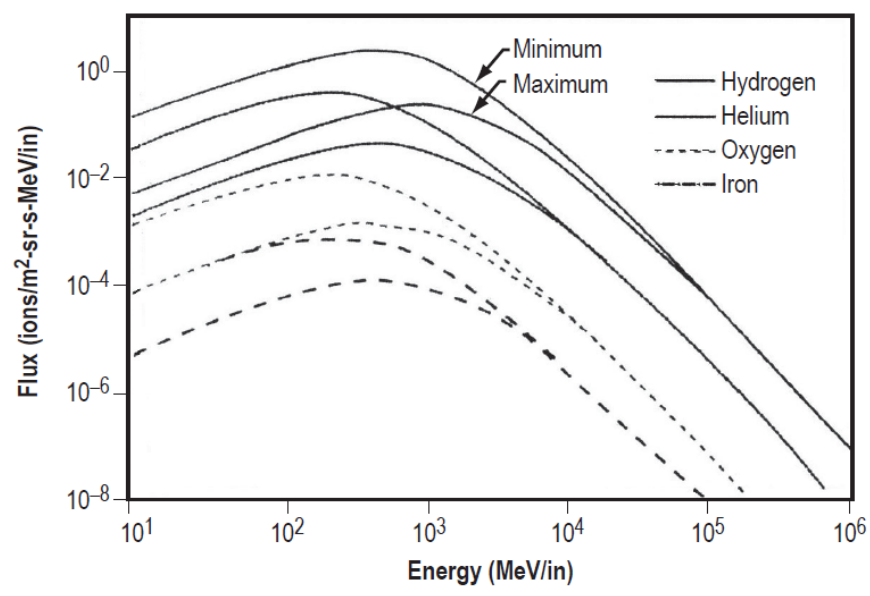

Figure 1 Differential spectrum of GCR at solar min and max (MeV/in is MeV per incident particle [14]. 
The Sun also contribute to the ionizing radiation. This radiation is due to Solar Particle Events (SPEs) or Solar Energetic Particles (SEPs). The energies of the SEPs are in average lower than energies of GCR particles but they are more abundant. The charged SPE particles are accelerated into the interplanetary space following mass ejections from the Sun corona. SPEs occur at unpredictable times. There is a correlation between SPE and the number of sunspots and thus the Sun activity [4]. SPEs occur about 5 to 10 times per year (except during solar minimum). It is hard to predict the exact onset time. It is only possible to tell whether a large or small SPE has occur many hours after the event [] $]$. Figure 2 illustrates proton fluence (in protons $/ \mathrm{cm}^{-2}$ ) of large SPEs with energies $\mathrm{E}>30,>60$, and $>100 \mathrm{MeV}$. Notice how the fluence of less energetic protons is higher than the fluence of higher energetic protons.

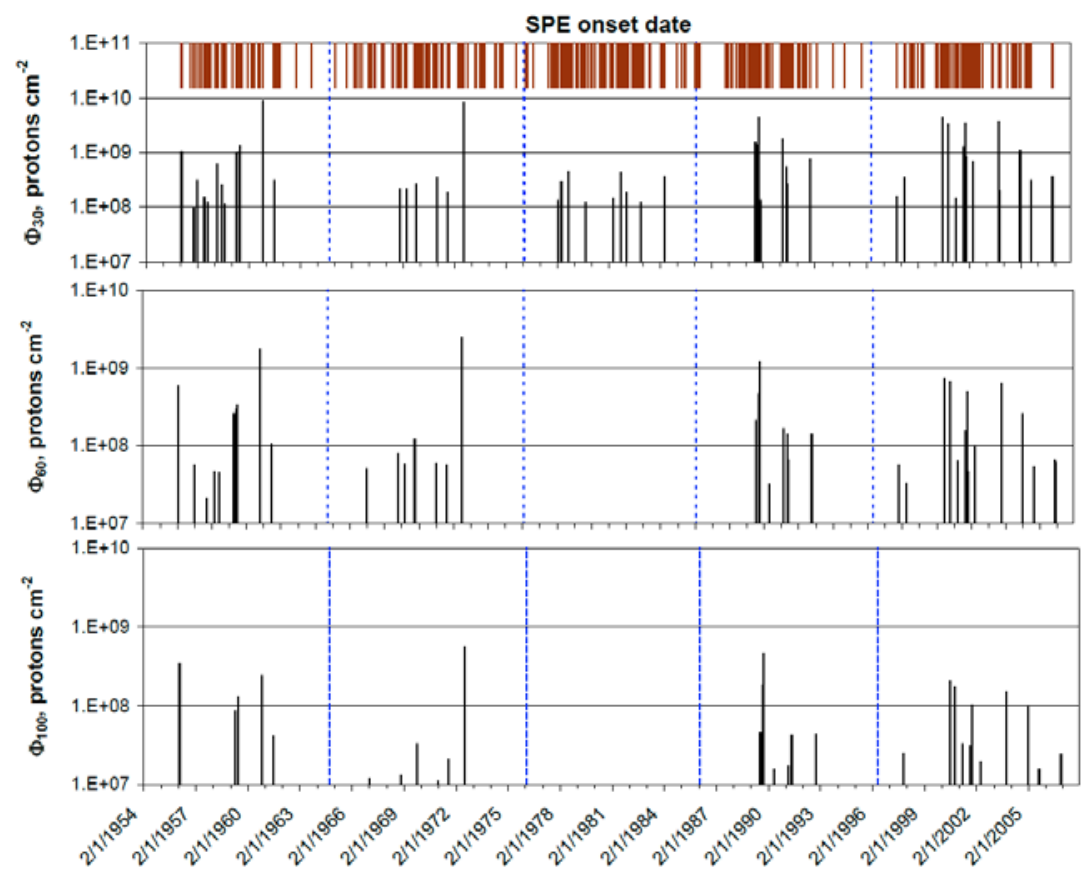

Figure 2 Large SPEs (proton fluence $>108 \mathrm{~cm}-2$ at E>30, 60 \& $100 \mathrm{MeV}$ ) as a function of time [13]

Like GCRs, SPEs primarily consist of protons but also include alpha particles and heavy ions with a composition that varies from event to event [1]. Protons have energies in the range of $1 \mathrm{keV}$ to $1000 \mathrm{MeV}$ but the main part of the spectra is below $200 \mathrm{MeV} / \mathrm{n}$; this is shown in figure 3. Some SPEs can reach a fluence of more than $10^{10}$ particles $/ \mathrm{cm}^{2}$, which happens in the timeframe from few hours to several days. For example, SPE of the August 1972 was potentially lethal for a human crew on the Moon surface without appropriate shielding [4]. While the average particle energy for SEPs is lower than for GCRs, the flux is much higher [1] 


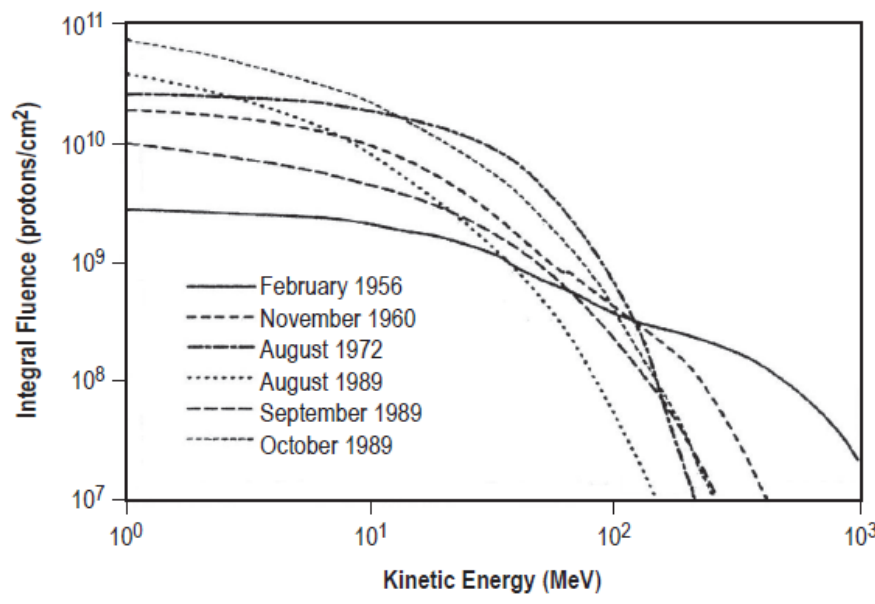

Figure 3 SPE integral spectra of larger solar particle events from 1956 to 1989 [1]

The Sun's activity not only drives SPEs but also affects the intensity of GCR. During their travel from the Sun, energetic particles interact with Galactic cosmic rays. This lead to reduction in the cosmic ray intensity, known as the Forbush Decrease (FDs). Decrease in intensity is followed by a slower recovery on a time scale of several days []ㅡ. SPEs are strong enough to affect GCR particle with energies less than about $2000 \mathrm{Mev} / \mathrm{amu}$, which are modulated by the 11-year solar cycle. The GCR intensity can drop by more than a factor of two during a solar maximum compared to a solar minimum when solar wind is the weakest [ $\underline{6}]$.

To understand the rest of the paper, one should be familiarized with basic concepts used in radiation protection

\subsection{Basic Concepts of Radiation}

Table 1 summarizes a few important quantities used in this paper. Radiation exposure is defined by the physical quantity called absorbed dose, $D$. It describes how much energy is absorbed by a unit mass. The units of absorbed dose are Joules $/ \mathrm{kg}$, which has a special name - Gray (Gy). An old equivalent to this unit is Roentgen, $R$, which had units of rad.

To quantify the health effect from the given amount of absorbed dose, a special quantity called Dose Equivalent is used. This is just dose absorbed, D multiplied by a scaling parameter called radiation quality factor, Q. Its units are called Sievert. The number of particles per unit area is called Fluence, F. It has units of $1 / \mathrm{cm}^{3}$. When particles pass through matter they lose energy at a certain rate, which depends on their kinetic energy and the charge-to-mass ratio of the material they traverse $\left(\mathrm{Z} / \mathrm{A}_{\mathrm{r}}\right)$. This rate has a special name - Linear 
Energy Transfer, LET. It has units of $\mathrm{keV} / \mu \mathrm{m}$. The relationship between dose fluence and LET is $D=F / \rho \cdot L E T$, where $\rho$ is density of the material.

Table 1 Important Radiation Quantities

\begin{tabular}{|c|c|c|c|c|c|}
\hline Quantity & Definition & Notation & Units (new) & Units (old) & Conversion \\
\hline Exposure & $\begin{array}{c}\text { Charge per } \\
\text { unit mass }\end{array}$ & - & - & Roentgen (R) & $\begin{array}{c}1 \mathrm{R}=2.58 \times 10^{-4} \\
\mathrm{C} / \mathrm{kg}\end{array}$ \\
\hline $\begin{array}{c}\text { Absorbed } \\
\text { Dose }\end{array}$ & $\begin{array}{c}\text { absorbed } \\
\text { energy by unit } \\
\text { mass }\end{array}$ & $D$ & Gray (Gy) & $\begin{array}{c}\text { Radiation } \\
\text { Absorbed } \\
\text { Dose (rad) }\end{array}$ & $\begin{array}{c}1 \mathrm{~Gy}=1 \mathrm{~J} / \mathrm{kg} \\
1 \mathrm{~Gy}=100 \mathrm{rad}\end{array}$ \\
\hline $\begin{array}{c}\text { Dose } \\
\text { Equivalent }\end{array}$ & $\begin{array}{c}\text { Biological } \\
\text { effect from } \\
\text { absorbed dose }\end{array}$ & $H$ & Sievert (Sv) & $\begin{array}{c}\text { Roentgen } \\
\text { Equivalent in } \\
\text { Man } \\
\text { (rem) }\end{array}$ & $1 \mathrm{~Sv}=100 \mathrm{rem}$ \\
\hline Fluence & $\begin{array}{c}\text { \# of particles } \\
\text { per unit area }\end{array}$ & $F$ & $1 / \mathrm{cm}^{3}$ & - & $D=F \cdot L E T / \rho$ \\
\hline $\begin{array}{c}\text { Linear } \\
\text { Energy } \\
\text { Transfer }\end{array}$ & $\begin{array}{c}\text { Rate of energy } \\
\text { loss }\end{array}$ & $L E T$ & $\mathrm{keV} / \mu \mathrm{m}$ & - & \\
\hline $\begin{array}{c}\text { Fluence } \\
\text { Spectra }\end{array}$ & $\begin{array}{c}\text { Spectra of } \\
\text { particles-to- } \\
\text { energy range } \\
\text { relationship }\end{array}$ & $\varphi_{j}(\mathrm{E})$ & $\begin{array}{c}1 / \mathrm{cm}^{3} \text { over } \\
\mathrm{MeV} / \mathrm{amu}\end{array}$ & & \\
\hline
\end{tabular}

As mentioned above, cosmic rays have a very broad energy range. Oftentimes it is useful to see how the abundance of specific type of particles spreads over this range. Figure 1 shows such spread for hydrogen, helium, oxygen and iron particles. Note that the energy range is given by energy per nucleon. This energy spectrum is denoted as fluence spectrum, $\varphi_{j}(E)$, where subscript $j$ refers to the particle type described by atomic and mass numbers []].

\subsection{Flux Types}

Both the GCR and SPE spectra are measured in terms of intensities of corpuscular radiation with various units that depend on detection method [12]. Intensity is a function of energy, time, steradians and area so it can get quite confusing. Usually there are two ways to specify intensity. The differential intensity, J(E) is the number of particles per unit time of a given energy within certain energy interval incident on a unit area perpendicular to the direction of observation. It has units of $\# / \mathrm{cm}^{2} / \mathrm{s} / \mathrm{sr} / \mathrm{MeV}$. Another way to describe intensity is with quantity called integral intensity, $\mathrm{J}(>\mathrm{E})$. The $>E$ means that the intensity is measured only for those particles whose energy is greater than the threshold energy. Integral intensity is just the differential intensity integrated over energy: 


$$
J(>E)=\int_{E}^{\infty} J(E) d E,
$$

and it has units of $\# / \mathrm{cm}^{2} / \mathrm{s} / \mathrm{sr}[\underline{12}]$. To complicate things even farther, since both $\mathrm{J}$ and $\mathrm{J}(>\mathrm{E})$ contain steradians units, they are called unidirectional differential and integral intensities respectively. If unidirectional intensities are integrated over $4 \pi$ steradians solid angle, they are called omnidirectional differential and integral intensities respectively:

$$
J_{\Omega}=\int_{0}^{4 \pi} J(\Omega) d \Omega,
$$

where $\Omega$ is solid angle.

Usually the term intensity is interchangeably used with the terms flux, $(J)$ or fluence $(\Phi)$. The difference between them is somewhat ambiguous. While in one source a flux is defined as a derivative of fluence with respect to solid angle, other sources express flux as a time rate of change of the fluence:

$$
\begin{aligned}
& J=\frac{d \Phi}{d \Omega} \\
& J=\frac{d \Phi}{d t}
\end{aligned}
$$

Using the first definition, a flux is the unidirectional intensity while fluence is omnidirectional intensity. Table 2 clarifies the complexity of these definitions.

Table 2 Difference Between Uni- and Omnidirectional flux a fluences

\begin{tabular}{|c|c|c|}
\hline w/ or w/o directional units & intensity type & unit \\
\hline unidirectional (flux) & differential & $\# / \mathrm{cm}^{2} / \mathrm{s} / \mathrm{sr} / \mathrm{MeV}$ \\
\cline { 2 - 3 } & integral & $\# / \mathrm{cm}^{2} / \mathrm{s} / \mathrm{sr}$ \\
\hline \multirow{2}{*}{ omnidirectional (fluence) } & differential & $\# / \mathrm{cm}^{2} / \mathrm{s} / \mathrm{MeV}$ \\
\cline { 2 - 3 } & integral & $\# / \mathrm{cm}^{2} / \mathrm{s}$ \\
\hline
\end{tabular}

In this study all the SPE spectra are given as omnidirectional fluences (usually differential) while the GCR spectra is given as an unidirectional flux because that is convention in the literature.

The plot of the differential or integral intensities versus energy is the most common way to depict the radiation flux of the GCRs and SPEs. Sometimes, instead of energy, the flux spectrum is given in terms of magnetic rigidity, $\mathrm{R}$ that is a measure of a particle’s resistance to a magnetic force that deflects it from a straight-line trajectory. These plots are usually reported by earth-based observatories because they measure particles with magnetic rigidity high enough to get through the "filter" of the earth magnetic field [12]. 


\section{SPECTRAL MODELS}

Sometimes, often during solar maximum, SPE fluences become extremely large. Such SPEs, called Ground Level Enhancements (GLEs), are of particular interest because of the acute radiation exposure they can cause to humans and electronics. GLE events are measured in terms of integral fluence. It is relatively easy to do. All it takes is to count the number of particles with each energy that hit a detector. Once the experimental data are collected, the spectrum must be determined by deriving a mathematical expression that has a good fit with the data acquired by observation. Today, scientific community uses three spectral models.

\subsection{Exponential Model}

The first method is exponential in proton rigidity fit (EXP) developed by W.R. Webber et al back in 1963. This method is an exponential function based on two proton integral data points at 30 and $100 \mathrm{MeV}$. Beyond $100 \mathrm{MeV}$ the particle energy spectrum is extrapolated to $1 \mathrm{GeV}$. As name suggest, EXP is a function of particle’s rigidity, not energy:

$$
\Phi(>E)=N_{0} \exp \left(-R / R_{0}\right)
$$

where $\Phi(>E)$ is the integral energy fluence in protons $/ \mathrm{cm}^{2} . \mathrm{N}_{0}$ is a normalization constant, $\mathrm{R}$ - proton rigidity in $\mathrm{MV}\left(10^{6}\right.$ volts $)$ and $\mathrm{R}_{0}$ - characteristic rigidity in $\mathrm{MV}$. The conversion from rigidity to energy in $\mathrm{MeV}$ is as follows:

$$
R=A / Z \sqrt{\left(E^{2}+2 E_{0 \mathrm{~A}} E\right)}
$$

where $A$ - atomic mass number, $Z$ - atomic number, $E_{0 A}$ - rest mass energy []], [12]. It is a usual practice to consider protons as the only constituent of SPE spectra. Therefore, $A, Z$ and $E_{0 A}$ are values for proton. EXP method was used for several decades until a new methodology was introduced.

\subsection{Weibull Model}

The second spectral fitting method is called Weibull fit, which is also an exponential function but unlike EXP it is exponential in energy:

$$
\Phi=\Phi_{0} \exp \left(-k E^{\alpha}\right)
$$

where $\Phi$ can be either the proton fluence or the proton flux having energy that exceeds a threshold energy $E$ [25]. Xapsos takes the units of $\Phi$ to be $\mathrm{cm}^{-2}$ when it represent integral fluence and $\mathrm{cm}^{-2} \mathrm{~s}^{-1} \mathrm{sr}^{-1}$ when it represents integral flux (see table 2). Three constants $\Phi_{0}, k$ and $\alpha$ are "Weibull” nonlinear regression fit 
parameters which are unique to each SPE. $\Phi_{0}$ is related to the event magnitude while $k$ and $\alpha$ are related to the spectrum hardness. The calculation method of these parameters is beyond the scope of this study and only parameters for notable solar events were adopted from the Xapsos paper and are listed in Table 3. The function is fitted to the maximum energy value of $1 \mathrm{GeV}$ [25].

Table 3 Weibull fit parameters [25]

\begin{tabular}{cccc} 
Onset Date & $\boldsymbol{\Phi}^{*}$ & $\boldsymbol{k}\left(\mathbf{M e V}^{-1}\right)$ & $\boldsymbol{\alpha}$ \\
\hline 4 Aug, 1972 & $2.455 \times 10^{10}$ & 0.0236 & 1.108 \\
12 Aug, 1989 & $1.622 \times 10^{11}$ & 1.166 & 0.4015 \\
29 Sep, 1989 & $3.631 \times 10^{10}$ & 0.877 & 0.3841 \\
19 Oct, 1989 & $1.23 \times 10^{12}$ & 2.115 & 0.2815 \\
23 Mar, 1991 & $1.66 \times 10^{11}$ & 0.972 & 0.4410 \\
${ }^{*}-\mathrm{cm}^{-2}$ (integral fluence) \\
$\mathrm{cm}^{-2} \mathrm{~s}^{-1} \mathrm{sr}^{-1}$ (integral flux)
\end{tabular}

\subsection{Band Function Model}

Finally, the third spectral model is called a Band function, which is a double power law in proton rigidity. The distinctive feature of this function is that it completely describes the entire proton energy spectrum. The method is based on the actual proton data observed in the range of medium to high energies (10 to several hundred $\mathrm{MeV}$ ). The proton spectrum at higher energies is deduced from the secondary neutrons produced by SPE protons colliding with the Earth's atmosphere, which are registered by high latitude neutron-monitor stations. This method is a function of proton rigidity. The integral fluence is broken into two parts depending on the proton's rigidity:

$$
\begin{array}{cc}
J(>R)=J_{0} R^{-\gamma_{1}} e^{-R / R_{0}}, & \text { if } R \leq\left(\gamma_{2}-\gamma_{1}\right) R_{0} \\
J(>R)=J_{0} R^{-\gamma_{2}}\left\{\left[\left(\gamma_{2}-\gamma_{1}\right) R_{0}\right]^{\left(\gamma_{2}-\gamma_{1}\right)}\right\} \cdot e^{\left(\gamma_{1}-\gamma_{2}\right)}, & \text { if } R \geq\left(\gamma_{2}-\gamma_{1}\right) R_{0}
\end{array}
$$

where $J(>R)$ is the integral fluence (particles $/ \mathrm{cm}^{2}$ ), $J o$ is the normalization constant, Ro is the characteristic rigidity $(\mathrm{GV})$ and $\gamma_{1}$ and $\gamma_{2}$ are spectral indices. The Band Fit parameters for several SPE events used in this study are included in Table 4. 
Table 4 Band Fit Parameters for Some SPEs Used in This Study [22]

\begin{tabular}{|lccc|cccc|}
\hline GLE's & & & \multicolumn{4}{|l|}{ Band Fit Parameters } \\
Date & & & Official No. & $\mathrm{J}_{0}(p / c m 2)$ & $\gamma 1$ & $\gamma 2$ & $\mathrm{R} 0(G V)$ \\
\hline 1956 & Feb & 23 & 5 & $8.79 \mathrm{E}+08$ & 0.584 & 5.04 & 0.3207 \\
1960 & May & 4 & 8 & $8.16 \mathrm{E}+05$ & 1.527 & 4.88 & 0.585 \\
1989 & Oct & 19 & $43 \mathrm{a}$ & $1.22 \mathrm{E}+09$ & 0.528 & 5.81 & 0.1621 \\
1989 & Oct & 19 & $43 \mathrm{~b}$ & $9.09 \mathrm{E}+09$ & 0.911 & 4.43 & 0.0844 \\
1989 & Oct & 22 & 44 & $1.09 \mathrm{E}+09$ & 1.226 & 7.25 & 0.1352 \\
1989 & Oct & 24 & 45 & $4.42 \mathrm{E}+07$ & 2.176 & 5.65 & 0.385 \\
1990 & May & 28 & 50 & $7.66 \mathrm{E}+07$ & 0.417 & 4.98 & 0.1433 \\
2005 & Jan & 20 & 69 & $3.80 \mathrm{E}+08$ & 0.719 & 5.78 & 0.204 \\
\hline
\end{tabular}

This method was successfully applied to about 70 GLE events that occurred after 1956. The Band function is a strong candidate for being the best method used for future GLE assessment and analysis [ $\underline{3}$ ]. 


\section{EFFECT ON HUMANS}

As mentioned earlier, the natural ionizing radiation environment is harmful to both electronics and crew. The effect on human health, however, is a major concern for the future manned mission developers. While in deep space, each cell of an astronaut's body would be hit by a GCR particle every few days []ㅡ. A good way to get an idea of what kind of radiation environment a potential astronaut would be subjected to, is to look at the data collected directly for interplanetary space. This data is now available from the Radiation Assessment Detector (RAD) on board of the Mars Science Laboratory (MSL) spacecraft that was launched to Mars in November 2011. Unlike similar instruments that are usually designed to measure primary (original) energetic particles and therefore placed outside a spacecraft, the RAD was located inside. Just as RAD, a human crew traveling to Mars inside a spacecraft would be exposed to a mixture of primary and secondary radiation. Therefore, the RAD data gives insight into the radiation environment inside a spacecraft carrying humans to Mars or other deep space destinations. The RAD has measured $1.84 \pm 0.33 \mathrm{mSv} / \mathrm{day}$. The MSL’s cruise to Mars took 253 days so the estimated total dose equivalent from both GCR and SEP events is $466 \pm 84 \mathrm{mSv}$ with only about 5.4\% of this value attributed to SEPs because the trip took place under conditions of low to moderate solar activity [26]. So how dangerous is $466 \pm 84 \mathrm{mSv}$ ?

To prevent risks that would jeopardize mission success and to limit chronic risks to acceptable levels, the space agencies of different countries have established so-called permissible exposure limits for radiation exposure of astronauts.

The radiation limits that NASA uses for exploration missions is as follows: career exposure to radiation should not exceed 3\% Risk of Exposure Induced Death (REID). The relationship between the level of absorbed radiation and the corresponding risk depends on many factors such as age, gender, duration since exposure, tissue types and others. Table 5 lists limits of career effective dose for a 3\% REID of 1 year missions. European, Russian and Japanese space agencies use somewhat modified recommendations for ground-based workers developed by the International Commission on Radiological Protection to establish their dose limits for astronauts. Table 6 lists radiation limits for these space agencies. 
Table 5 Example career Effective dose limits for 1-year missions

for a 3\% REID [7].

Career Effective Dose (mSv) for a 3\% REID

\begin{tabular}{ccc}
\hline Age at Exposure (years) & Males & Females \\
30 & 620 & 470 \\
35 & 720 & 550 \\
40 & 800 & 620 \\
45 & 950 & 750 \\
50 & 1150 & 920 \\
55 & 1470 & 1120
\end{tabular}

Now it is possible to evaluate the dose equivalent value obtained by the RAD with the NASA dose limits. Suppose a potential mission performs a round trip to Mars during the same conditions of low to moderate solar activity. The total dose the crew would get is about $932 \mathrm{mSv}$. This is close to the limit for a 45 years old male. In addition, if landing is involved, exposure during the time spent on surface of Mars also has to be added. Since the wait time for the next launch window may take a year, this additional dose can be quite significant. Therefore, a shielding with radiation attenuation properties more efficient than the typical manned spacecraft must be developed in order to make human presence safe or even possible in interplanetary space.

Table 6 Radiation Limits for Other Space Agencies [7]

\begin{tabular}{|c|c|c|}
\hline \multirow{2}{*}{ Limit } & \multicolumn{2}{|c|}{ Value (mSv) } \\
\hline & ESA & RSA \\
\hline Career & 1000 & 1000 \\
\hline $\begin{array}{l}\text { Blood Forming } \\
\text { Organs (BFO) }\end{array}$ & $\begin{array}{l}250 \text { for } 30 \mathrm{~d} \text {; } \\
500 \text { for annually }\end{array}$ & $\begin{array}{l}150 \text { for acute (1-time) } \\
250 \text { for } 30 \mathrm{~d} \\
500 \text { for annually }\end{array}$ \\
\hline Eye & $\begin{array}{l}500 \text { for } 30 \mathrm{~d} \text {; } \\
1000 \text { annually }\end{array}$ & $\begin{array}{l}500 \text { for } 30 \mathrm{~d} ; \\
1000 \text { annually } \\
2000 \text { for career }\end{array}$ \\
\hline Skin & $\begin{array}{l}1500 \text { for } 30 \mathrm{~d} \\
3000 \text { for annually }\end{array}$ & $\begin{array}{l}1500 \text { for } 30 \mathrm{~d} \\
3000 \text { for annually } \\
6000 \text { for career }\end{array}$ \\
\hline
\end{tabular}




\section{PRINCIPALS OF HIGH ENERGY PARTICLE INTERACTION WITH MATTER}

It is unlikely that the shielding approach can provide a technological solution that is feasible today because of the very high energies that GCR particles can reach and because of very high launch costs caused by increasing the amount of shielding material required for significant mitigation properties.

Materials with the smallest mean atomic mass are usually the most efficient shields for both SPEs and GCRs. When particles traverse a structural material, they interact with the nuclei of that material and therefore lose energy. Another consequence is a change in the composition of the radiation field or particle fluence. These changes in energy and fluence depends on the material that particles traverse. More specifically - the number of atoms per unit mass in the traversed material. The energy loss by ionization of a single component of shielding material with atomic number $Z$ is proportional to the number of electrons per atom and thus proportional to $Z / A$, where $A$ is the atomic mass number $A$ of each element of the material. The energy lost per gram of material and per incident fluence (e.g., in units of particles per $\mathrm{cm}^{2}$ ), the "mass stopping power,” is also inversely proportional to the density, $\rho\left(\mathrm{g} / \mathrm{cm}^{2}\right)$ of the material, so that the energy lost by one incident particle per $\mathrm{cm}^{2}$ per unit mass is proportional to $\mathrm{Z} /(\mathrm{A} \rho)$ [6].

This ratio consists of two important components. The first component is Z/A ratio. It is proportional to the number of electrons per nucleon. Materials with small atomic mass have the highest number of electrons thus the ratio is higher. Hydrogen, for example, has the highest number of electrons per nucleus with Z/A ratio of 1 .

The second component is density. The smaller the density, the higher the ratio. Therefore, the energy lost by energetic particle is higher for low density materials with small atomic mass numbers. Liquid hydrogen should be the most efficient material. Figure 4 shows values of $\mathrm{Z} /(\mathrm{A} \rho)$ for different materials.

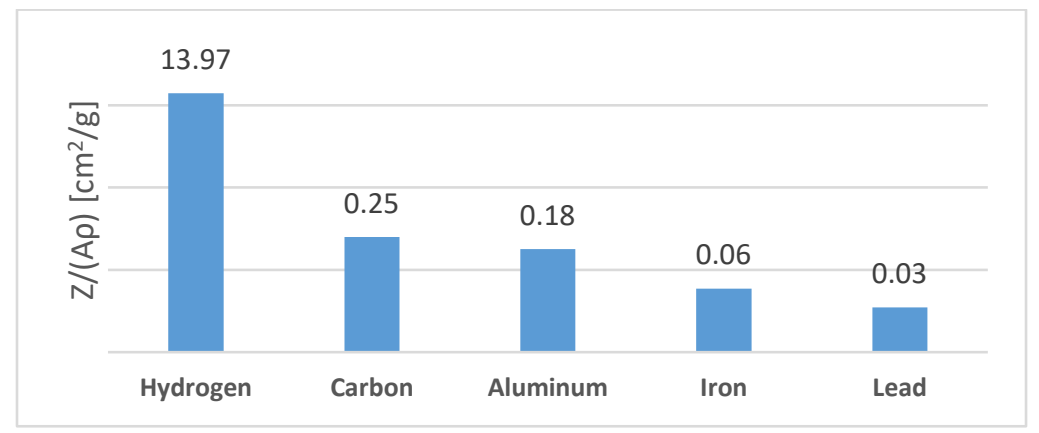

Figure 4 The Z/(A $\rho)$ Ratio Values for Different Materials (Hydrogen is in liquid form) 
When an energetic particle interacts with an atom of the shielding material, both split into pieces producing secondary nuclei. These secondary particles are important in shielding considerations. Some elements break into neutrons while others, such as carbon, break into three $\alpha$-particles. Although $\alpha$-particles are much more biologically damaging, neutrons are of higher concern because of their longer ranges. Energetic particles lose their energy through ionization of target atoms. If this energy is greater than 1000 $\mathrm{MeV} / \mathrm{amu}$, ionization processes release electrons energetic enough to cause further ionization of nearby atoms and these electrons, having energies more than $1 \mathrm{MeV}$, are called $\delta$-rays. The lateral spread of $\delta$-rays is called track-width, which depends on velocity (energy) of the original particle and its atomic number according to the following ratio: $(\mathrm{Z} / \beta)^{2}$, where $\beta$ is the particle velocity scaled to the speed of light. Figure 5 clearly showing the increasing lateral spread of $\delta$-rays along the track with increasing the charge Z [6].

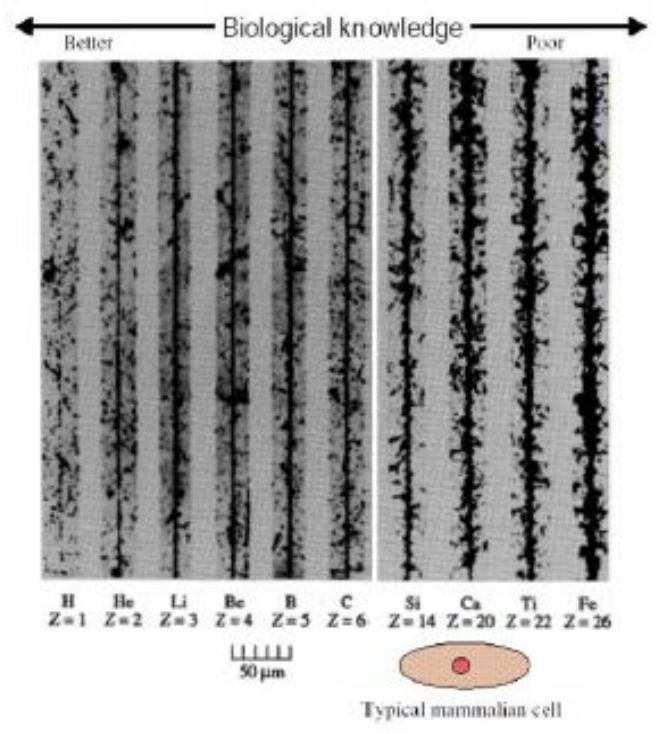

Figure 5 Different ions tracks in nuclear emulsion. Note the increase in number of $\delta$ rays along the track with increasing atomic number [6]

On one hand, low-Z particles have a higher biological effectiveness. On the other hand, higher $\mathrm{Z}$ nuclei at the same LET (see table 1) affects more cell layers before it deposits all of it energy. To compare the biological effect of different particles types, a special term called Relative Biological Effectiveness, RBE is used. RBE is a ratio of doses causing identical effect. The numerator of this ratio is the dose due to wellstudied gamma or X-rays and the denominator is the dose due to the particle being studied. RBE data is used to make estimates for human risk by defining a radiation quality factor. For terrestrial radiation exposures, 
quality factors, Q are determined uniquely by LET. For example, Q=1 corresponds to LET of $10 \mathrm{keV} / \mu \mathrm{m}$ while $\mathrm{Q}=30$ corresponds to $100 \mathrm{keV} / \mu \mathrm{m}$. This convention is not needed for space radiation environments and quality factors are then defined by E and Z instead of LET. Secondary particles, which are produced when primary particle traverse shielding material, can have quality factors higher than the primary particle. Figure 6 illustrates the dependence of the radiation quality factor for solid cancer on the primary particle's energy and Z. This figure illustrates the complexity of GCR interactions with matter. For example, consider a Fe particle with an energy of above $800 \mathrm{MeV} / \mathrm{amu}$. While traversing through the shielding material, it loses energy, which can be illustrated by following the Fe curve from the right vertical line to the left. The quality factor in this case increases. If, on the other hand, the initial energy of the Fe particle is below $500 \mathrm{MeV} / \mathrm{amu}$, the loss of energy causes quality factor to decrease.

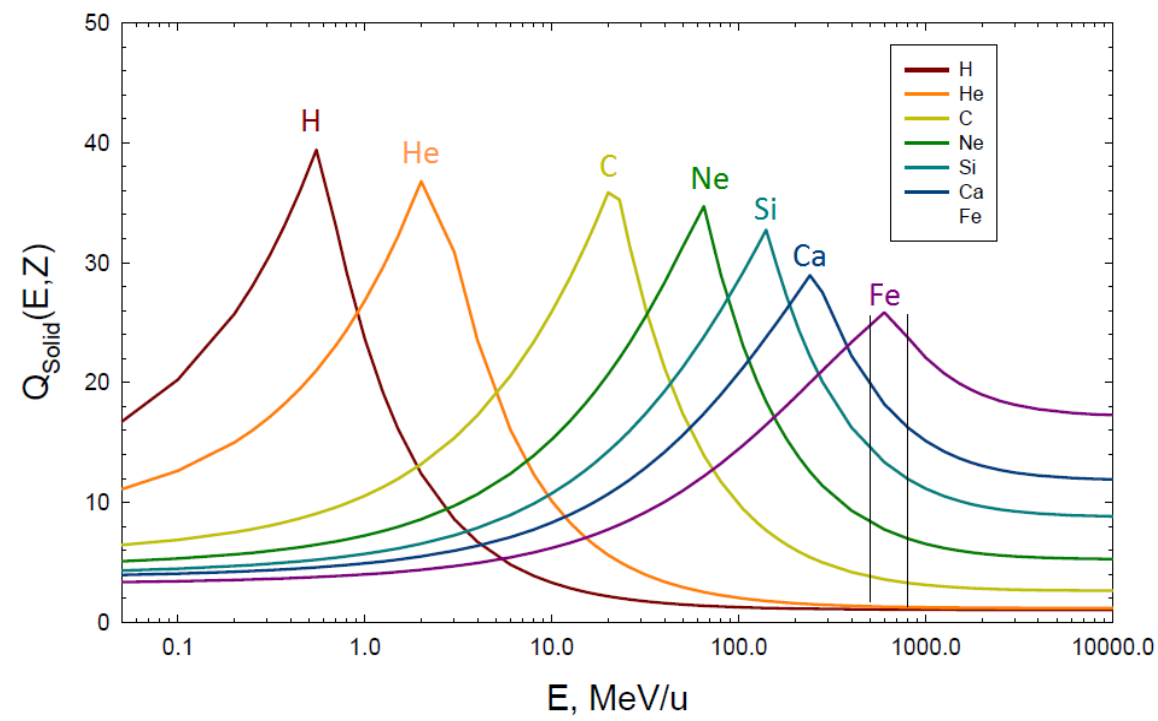

Figure 6 Dependence of the quality factor on a particle's energy for several GCR nuclei [ 6$]$

Another example that illustrates the complexity of the problem is a fragmentation of a Fe particle which creates new particles with lower $\mathrm{Z}$ and $\mathrm{E}$ as well as high energy neutrons, protons and other light particles. That increases population of the radiation field. Therefore, it is important to define particle flux spectra to evaluate effectiveness of shielding materials [ 6$]$. 


\section{TRANSPORT CODES}

To predict the effectiveness of shielding materials there are two options. The first, is to reproduce the radiation environment and make measurements behind the shield. Unfortunately, GCR particles can reach very high energies, even higher than the Large Hadron Collider - today the most powerful particle accelerator - can achieve not to mention the very high cost of using it anyway. Such high-energy ions, however, may have a noticeable contribution to the overall absorbed dose due to GCR. Therefore, it is impractical to reproduce the GCR environment in a laboratory on Earth and it is too expensive to perform such experiment in space every time a new shielding needs to be tested. The second option is to evaluate effectiveness of shielding materials by modeling the space radiation environment using a transport code. Such software is applied in many fields to design detectors, accelerator shielding, dosimetry, and many others. Transport codes characterize the modified radiation field downstream of the point of interaction between the incident radiation and the target nuclei. This characterization is in terms of absorbed dose or dose equivalent which is necessary to assess the response of electronics or, more importantly, biological systems [2] ]. Every transport code utilizes one of the two methods: analytic and probabilistic (Monte Carlo method). Analytic methods compute a mathematical function, which have a unique value for any input on its domain. Probabilistic or Monte Carlo (MC) methods rely on repeated random sampling to obtain numerical results. The histories of particle interactions are simulated using random numbers that model the probability of particle interactions. One example of deterministic transport code is HZETRN developed by NASA Langley Research Center. A special online tool called OLTARIS is used to provide this code in a user-friendly environment. NASA engineers use this code to evaluate dosimetric information required to design space vehicles. It is based on one-dimensional formulation of the Boltzman transport equation [24]. Examples of MC codes are MCNPX developed by Los Alamos National Laboratory, PHITS, developed by several institutes in Japan and Europe. Another example of a MC code is FLUKA. Since this code is used in this paper, it requires a bit more detailed introduction. FLUKA is a product of European Organization for Nuclear Research (CERN). This fully integrated Monte Carlo simulation package simulates particle transport and interactions with matter. Fluka's range of application is quite broad, spanning from accelerator shielding to dosimetry, radiotherapy and others. It can simulate interactions of about 60 different particles with the energies up to $20 \mathrm{TeV}$. The code can only be used with Linux and requires g77 compiler to build and run the user programs [1ㅣ]. The Fluka Advanced Interface 
(FLAIR) is a convenient graphical user interface to run FLUKA. It is an input file editor, which inspects the input syntax for errors and flags incorrect entries. 


\section{FLUKA}

\subsection{How FLUKA Works}

FLUKA's input is a text file that has a list of option lines called cards. Cards consist of six numerical quantities (e.g. energy, coordinates, etc.) called WHATs where most of the information required for a card to serve its purpose is placed. Such information may be numerical data such as coefficients, energy ranges or dimensions. Every card belongs to a certain category. For example, category Geometry deals with the bodies and regions, Scoring contains detector cards and so on. Scoring cards need to be explained in more details because they are important for the further discussion. Each scoring card is designed to detect a specific quantity. The two cards used in this study are USRBDX and USRBIN. The first one defines a detector for a boundary crossing fluence estimator. It is used to score the differential fluence of the source and secondary particles in a shield. The output of this card is flux integrated over solid angle (omnidirectional) and has units of ions $/ \mathrm{cm}^{2} / \mathrm{GeV} / \mathrm{pr}$. The second scoring card, USRBIN, is used to score absorbed dose or dose rate. The output of this card is given in the units of $\mathrm{GeV} / \mathrm{g} / \mathrm{pr}$. It describes how much energy was absorbed per unit primary weight [10]. The fluka's output is always expressed “per primary particles” (hence the “pr” in the output units). The physical meaning of this primary varies with the type of source being simulated. In the case of a SPE source, the primary is a total fluence, sort of a normalization value that can be found analytically by calculating the area under the differential fluence and then employing the first fundamental theorem of calculus:

$$
\text { primary is: } \quad \Phi_{\text {total }}=\Phi\left(E_{\max }\right)-\Phi\left(E_{\min }\right)
$$

where $\Phi$ is the integral energy fluence in $\# / \mathrm{cm}^{2}$ (or primary/ $\mathrm{cm}^{2}$ for unit conversion purposes). The calculated primary is then applied to the output of both scoring cards: USRBIN and USRBDX. It will be shown further that, in the case of a GCR source, the physical meaning of a primary is related to time. Notice, however, that in the case of an SPE source, the units of both scoring cards does not contain time. Time doesn't show up at all in the case of SPE source while it does appear in the primary value when the source is a GCR. This is because, in the case of a SPE, the source is defined as an event which lasted and was recorded for a certain amount of time in the past. As a result, when the source is defined as SPE event, USRBIN detector measures absorbed dose instead of dose rate as in case of GCR source. This time span is embedded in the SPE source.

FLUKA uses a special user routine code to read from the source file and to generate particles in the amount and of the energy specified in the source file. When these particles are incident on a scoring detector, 
the output of the detector card is always time independent because again the source is a finite event rather than a constant radiation background in the case of a GCR source while in a case of SPE, time is embedded in the primary value. Theoretically, both the source input and the fluence of the particles generated by fluka should be identical. This was checked and will be discussed farther in this paper. Also, the exact way a FLUKA source was created will be explained later.

When the source is defined as GCRs, the time does appear in the output. The GCR source, however, was not modeled as an event but rather as a continuous background radiation. Therefore, it is time dependent and, as mentioned earlier, the time unit of the scoring card is embedded in the normalization factor. However, this factor in the case of GCRs is defined differently than in the case of a SPE source. Instead of calculating the normalization factor from the source fluence, it can be found from the fluka output data. Fluka has a build in GCR package which not only generates a source for the fluka's run but also provides two values that are used to calculate the normalization factor and one of these values is time dependent. The GCR package is described later on.

Several input parameters affect the quality of the fluka output. One is the number of requested events also known as the number of source history particles in other codes. This parameter specifies how many particles are generated. More particles mean more points in the output, which makes the output more meaningful. However, a large value for this parameter comes with a price of a long FLUKA run: the greater the value, the longer the process. The second parameter is the number of runs. This value affects the output uncertainty. Obviously, the more times the same experiment is repeated, the smaller the statistical uncertainty [10].

\subsection{Geometry}

The traditional approach of transport codes to implement a geometry based on a Constructive Solid Geometry (CSG) which involves the boolean geometry tree - a hierarchical structure of the geometry elements. The basic idea is that any geometrical objects, regardless of their complexity, are made of elementary shapes (primitives) that can be added to or subtracted from one another to produce complex objects [21]. Figure 7 demonstrates how CSG works. A combination of objects are joined into a region. A material is then applied to a region, not to an object. Each region can be made of only one material. Therefore, FLUKA's particles interact with regions, not objects itself. For example, it is mandatory to assign a special material called blackhole to a region where all particles vanish once they reach this region. Blackhole serves as a particle 
terminator because it has an infinite absorption cross section. For all fluka experiments performed for this paper, such a region was a sphere, which encompassed all other regions.

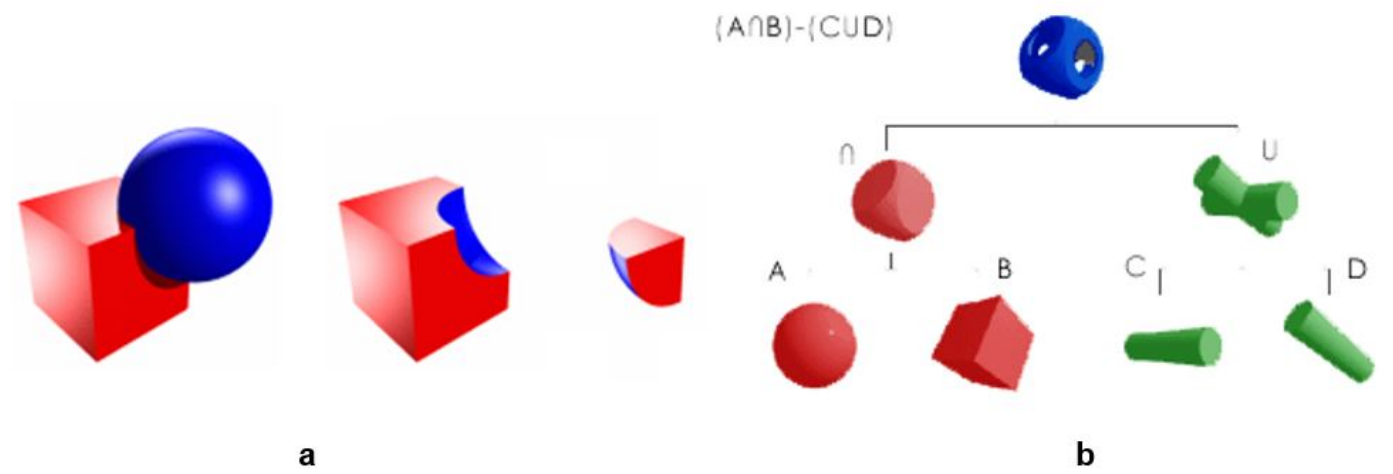

Figure 7 (a) Constructive Solid Geometry, (b) example of a boolean geometry tree. Courtesy of N. Stewart

\subsection{Creating a Source}

Two source types were utilized for this study: GCR and SPEs. The GCR source is a part of a package build into FLUKA. This package contains a model of the energy spectrum and composition of cosmic rays and the local interstellar medium. Ion composition of the galactic flux has been produced by the modified Badhwar code for various modulation parameters and written on 28 “.spc" files (Z+<PhiMV $>+$.spc) [18]. Each file corresponds to an element from Hydrogen to Nickel. There are two groups of 28 files:

- Solar minimum: <zzphi0465.spc>

- Solar maximum: < zzphi1440.spc >

Each file contains a differential flux in ions/ $\mathrm{cm}^{2} / \mathrm{s} / \mathrm{sr} /(\mathrm{MeV} / \mathrm{amu})$ with corresponding energy bin in MeV/amu, where $s r$ is steradians and $a m u$ is atomic mass number. The spectrum is modified to follow recent data sets from the AMS and BESS experiments up to $100 \mathrm{GeV}$, according to the so-called ICRC2001fit [18].

To set up the GCR source in FLUKA, one specifies an energy interval and choose a starting radius (radius of the emission sphere in case of spherical geometry). The GCR package in FLUKA is designed mostly to model the interaction of energetic particles with Earth's atmosphere. All necessary normalization factors for different layers of the atmosphere are predefined. However, the purpose of this paper requires an interplanetary GCR source. Fortunately, all it takes to ignore these atmosphere-related normalization factors is to choose the "NO-NORM" in the GCR-SPE card. This way one obtains a raw GCR data without any kind of further 
normalization [18621]. Figure 12a shows the GCR fluence of four energetic particles used in GCR package with one correction: instead of $\mathrm{cm}^{2}$, one should read $\mathrm{m}^{2}[\underline{20}]$.

The normalization factor in case of the raw GCR source can be calculated based on the two values provided by GCR package that can be found in the output file (.out), in the section called "Output during Transport.” These two values are Global Normalization (integral over energy and angle) called Fluxst and Equivalent Flux called Flux in the output file. The first value depends on the number of source ions chosen to be included into the source (number of .spc files involved) and has units of ions $/ \mathrm{cm}^{2} / \mathrm{s}$. Notice the time unit appears in the normalization factor. The second value, the Flux, depends on the radius of the emission sphere and has units of $\mathrm{part} / \mathrm{pr} / \mathrm{cm}^{2}$, where pr is primary mentioned earlier. The Flux value is basically the isotropic flux exposure to galactic cosmic radiation during a solar minimum divided by the surface area of the emission sphere. For the solar minimum, this flux equals to about 4 protons $/ \mathrm{cm}^{2} / \mathrm{s}$ [12]. A normalization factor is found by dividing Fluxst by Flux. The result is a quantity with units of $\mathrm{pr} / \mathrm{s}$. Therefore, multiplying this quantity by the USRBIN output (Gy/pr after some conversion), one should get the absorbed dose rate.

The SPE source, in this study, was given as an ASCII (text) file containing energy data in discrete intervals with corresponding differential fluence values. This file is generated uising MATLAB code by vectorising differential form of one of the spectral fitting equations such as equations (5) - (9). The output of this code is an $\mathrm{n} \times 2$ matrix where $\mathrm{n}$ is the number of discrete energy bins (usually 2000) and two columns are energy and differential fluence. FLUKA reads this file using a special user routine algorithm coded in C. 


\section{FLUKA RESULT VERIFICATION}

\subsection{FLUKA Validation By Repeating The Aghara Paper Experiment}

Before FLUKA can be used to study shielding characteristics of different materials, it is important to validate its results. This verification was performed by repeating a simple experiment described in a published and peer reviewed paper and by comparing results. The paper used in this study was written by S.K. Aghara et.al [2]. This paper investigates the impact of several SPE fluxes transported through an aluminum shield followed by water. The simulation was performed by three transport codes: MCNPX, PHITS and OLTARIS. This paper reports two results that are of main interest for the purpose of FLUKA validation: fluence spectra of four SPE events and the dose distribution that these events cause in the target. The same setup used in this paper was repeated with FLUKA. The following is the description of two main elements of this setup: the source and the target.

\subsubsection{The Source}

The source in Aghara paper are four historically significant SPE events that occured in Feb 1956, Aug 1972, Oct 1989 and Mar 1991. Two things should be clearly defined before an SPE source is simulated by a transport code:

1. the function form (integral or differential fluence)

2. particular fitting function (EXP, Weibull or Band)

All events in this experiment were modeled only as proton fluence. The first event in the Aghara paper is called 56 Webber after W. R. Webber who used the EXP method, eq (5), to model the spectrum of this event back in 1963. The proton intensity is given in the differential form:

56 Webber

$$
\frac{d \Phi}{d E}=1.09 \times 10^{8} \frac{E+938}{\sqrt{E(E+1876)}} e^{\left(\frac{\sqrt{E(E+1876)}}{100}\right)}
$$

Note that this expression is obtained from eq. (5) by expressing rigidity in terms of energy according to eq. (6) and differentiating with respect to energy with $\mathrm{N}_{0}=1.09 \times 10^{10}$ and $\mathrm{R}_{0}=100$.

It is not clear which fitting model was used for the 1972 event in the Aghara paper. It should have been developed by the Langley Research Center since it is referred as 72 LaRC. Its differential form is: 


$$
\frac{d \Phi}{d E}=2.2 \times 10^{7} e^{\left(-\left(\frac{E-100}{30}\right)\right)}
$$

This is not the differential form of Weibull function should look like and it is also not the double power law of the Band function. Therefore, it should be some form of the EXP function.

The 1989 and 1991 events are both modeled by a Weibull function. The first is referred as 89 Weibull and the second one as 91 Carr. Its nonlinear regression fit parameters are given in Table 7. The differential forms of these events are as follows:

89 and 91 Weibull

$$
\frac{d \Phi}{d E}=\Phi_{0} \kappa \alpha \cdot E^{\alpha-1} e^{\left(-\kappa E^{\alpha}\right)}
$$

The source is modeled as a pencil beam originated from a point located a certain distance from the target along Z-axis [2]

It is important to specifically define the source in a transport code in order to perform an accurate normalization. This means that, the source flux should be defined in either differential or integral form i.e. $\mathrm{J}$ or $\mathrm{J}(>\mathrm{E})$. FLUKA requires the source to be defined in differential form because this form doesn't change with a choice of energy step which the user is allowed to vary. If a source depends on the energy step, the magnitude of the result may vary with the choice of the energy step size. Differential flux, however, is by definition normalized by energy and is thus independent of the energy step size.

Table 7 Spectral Weibull parameters for two SPE events [2]

\begin{tabular}{|c|c|c|c|}
\hline Event & $\boldsymbol{\Phi}_{0}$ & $\boldsymbol{\kappa}$ & $\boldsymbol{\alpha}$ \\
\hline October 1989 (89 Weibull) & $7.323 \times 10^{11}$ & 2.115 & 0.2815 \\
\hline March 1991 (91 Carr) & $1.47 \times 10^{12}$ & 0.972 & 0.4410 \\
\hline
\end{tabular}

\subsubsection{The Detector}

The detector in the FLUKA simulation is modeled as a disk oriented normal to the flux of the incident particles. Vacuum is assigned to the detector region. This region is “floating” inside the Void region made also of vacuum which in turn is surrounded by a blackhole region. The detector's radius is $15 \mathrm{~cm}$ and its thickness is $0.5 \mathrm{~cm}$. The detector thickness doesn't actually matter since it is a border between two regions (void and detector) which is used to measure the fluence. In fact, a separate region to score fluence is redundant because the border between the void and the target can be used as a detector. The reason to add an additional region is to accelerate the fluka run. The target is made of materials other than vacuum and they are computationally 
expensive. Therefore, suppressing the target and leaving only a "simple" vacuum detector allowed a high quality result to be acquired within a short timeframe.

\subsubsection{The Target}

An important quantity in the absorption of radiation called area density is a common way to measure thickness of a shield. The definition of area density is mass per unit area of a two-dimensional object. Area density is basically the shield's thickness times the density of the material of which shield is made:

$$
\text { A.D. }=\rho \cdot t
$$

Area density is an intermediate step in conversion between thickness and mass of material behind given area and since dose is energy absorbed by a given mass, area density is more convenient than thickness.

The target is a cylindrical body that consist of two parts: aluminum shield followed by a water slab (fig 8). The Shield has either a thickness of 10 or $20 \mathrm{~g} / \mathrm{cm}^{2}$. This is a typical average wall thickness of the International Space Station and the Space Shuttle [2]. The water is $30 \mathrm{~g} / \mathrm{cm}^{2}$, which corresponds to the thickness of an average human body. In fact, there is a negligible difference in stopping power between water and tissue therefore water is commonly used as tissue equivalent.

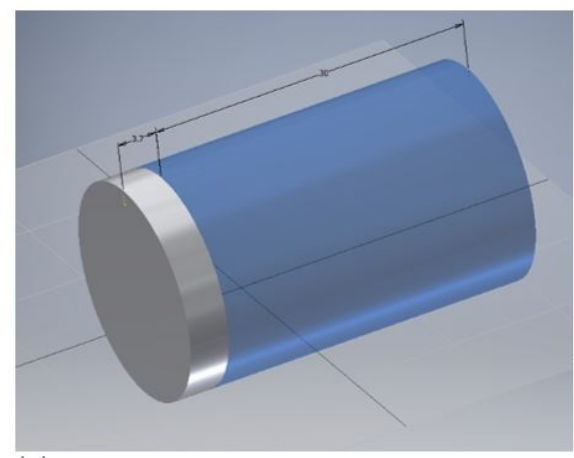

(a)

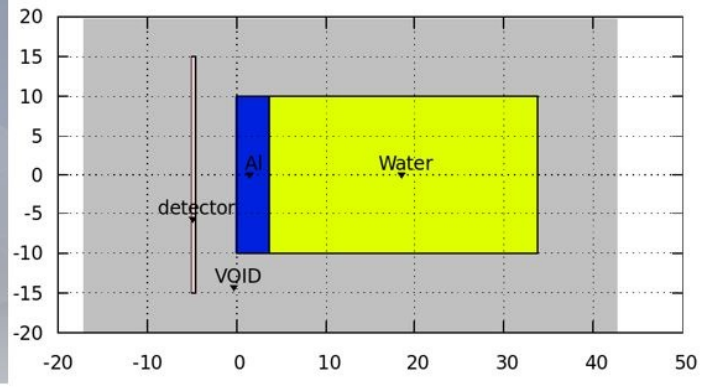

(b)

Figure 8 Target from the Aghara paper. (a) 3D model, (b) how it is modeled in FLUKA

\subsubsection{Results Comparison}

The FLUKA validation consist of two goals:

1. Make sure the proton fluence generated by FLUKA is identical to the source fluence and matches the fluence reported in the paper 
2. Make sure that the dose distributions within the target due to all four SPE events are in good agreement with the paper results

The criterion for good agreement is determined in the following way. Both fluence reported in paper and fluence generated by FLUKA are plotted. The agreement is good if the paper's fluence lies within error bars of the FLUKA's fluence along most of the energy spectrum.

The first goal was achieved as follows: after the above equations of differential fluence where converted into vector form and a source file for each event was generated by MATLAB code, the FLUKA's user routine read it and a simulation of proton fluence was generated by FLUKA. This simulated proton fluence was measured by the USRBDX detector. It is important to understand that there are three values, which theoretically should be identical:

- $\quad$ Fluence reported in the Aghara paper

- Fluence constructed from equation

- Fluence scored by detector

All three were plotted for four SPE sources to determine how well was the USRBDX measurement. This comparison is shown in Figure 9, which contains four proton fluence spectra as they appear in the Aghara paper. It also shows how well the FLUKA simulation matches the source equation and how well both (FLUKA and equation) match the spectra reported by Aghara's paper. It contains an insert showing the regions with the worst agreement for the $72 \mathrm{LaRC}$ and 89 Weibull models. These inserts allow to distinguish the analytic curve from the paper and makes it possible to see small differences in the FLUKA output. Even within the worst region the theoretical fluence (generated by eq.12) lies within the error bars of the fluence generated by FLUKA. The fluence reported in the Aghara paper (dashed line) was obtained by digitizing the plot found in that paper and thus has an additional uncertainty margin. Although figure 9 does not show this margin, it is seen that the curve lies close to the theoretical values - almost touching the FLUKA's error bars. For that reason it was decided that it is safe to assume a good agreement between all three aforementioned values. 


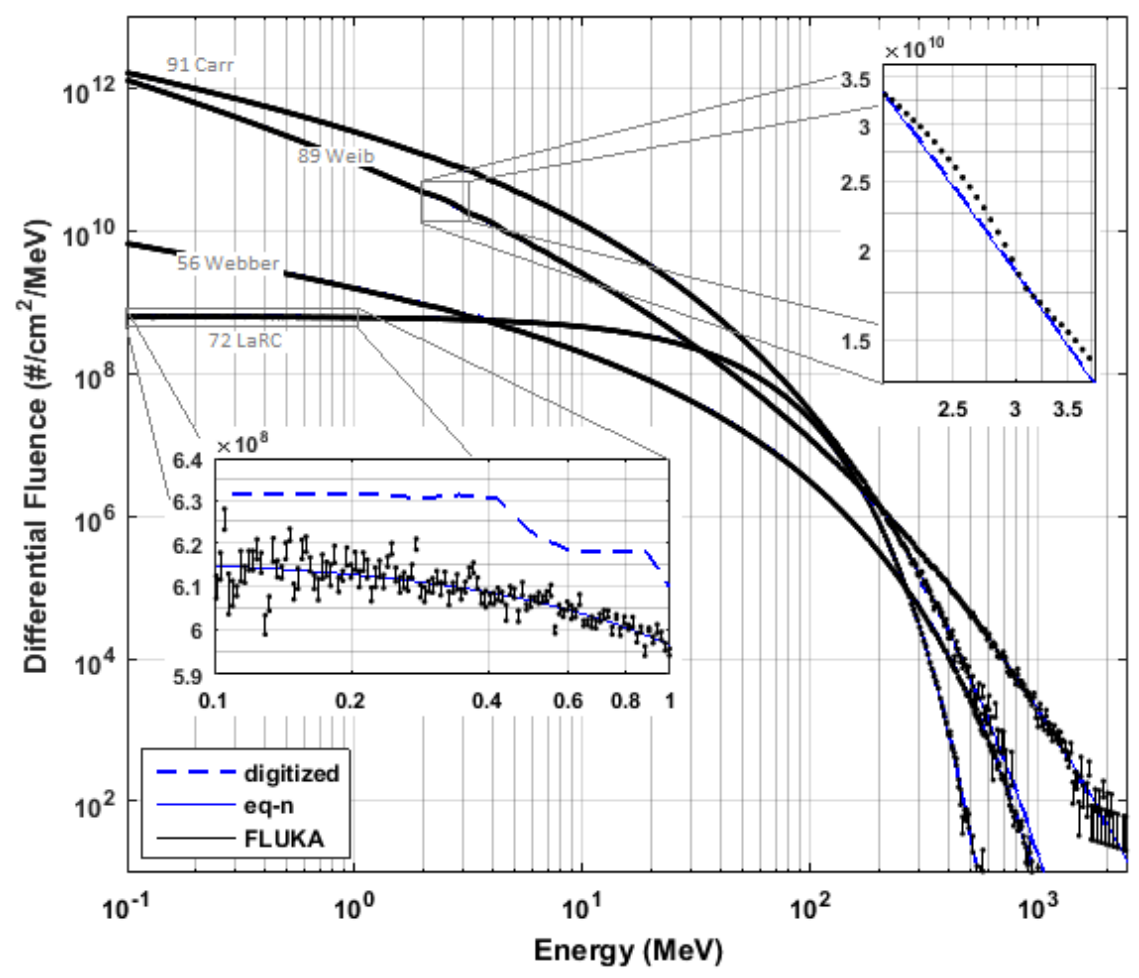

Figure 9 Fit of the FLUKA-generated Fluences with Fluences from the Aghara's Paper and Theoretical Fluences

In order to quantify the difference among these results a method of Mean Square Deviation (MSD) is utilized [2]:

$$
M S D=\frac{1}{n}\left(\sum\left(\theta_{i}-\theta_{i}^{\prime}\right)\right)
$$

where $\theta$ and $\theta^{\prime}$ are values of fluence at energy $i$ and $n$ is the total number of the fluence data points. This value is then normalized by the highest fluence $\theta_{\max }$. After MSD is normalized by the highest fluence, the resultant value becomes unitless and by itself provides little information except that the smaller number of MSD indicates a smaller difference. These values are useful when they are compared between each other and give a convenient way to judge how close one curve is to the other. Table 8 shows MSD values that compare FLUKA fluence with digitized and theoretical fluences. It is clear that the FLUKA result has much better agreement with the theoretical values than the digitized curve from Aghara's paper. Also, it is clear that both 56 Webber and 72 LaRC have better agreement by an order of magnitude compared to 89 Weibull and 91 Carr. The exact reason is not clear but it should be related to the mechanism FLUKA generates the source fluence. 
Table 8 MSD Values* for Fluxes Obtained by FLUKA, with eq.15 and from Aghara's Paper

\begin{tabular}{|c|c|c|}
\hline & FLUKA Result vs Digitized & FLUKA Result vs Theoretical $^{*}$ \\
\hline 56 Webber & 4.22 & 0.12 \\
\hline 72 LaRC & 2.01 & 0.04 \\
\hline 89 Weibull & 159.15 & 19.8 \\
\hline 91 Carrington & 86.63 & 20.64 \\
\hline
\end{tabular}

- \#/1E5

Figure 10 shows percent uncertainties for all four sources. Notice that the uncertainty in USRBDX output increases significantly with increasing in energy reaching $100 \%$ at the end of spectrum. This is because protons having high energy are less abundant than those with lower energy so when fluka simulates a spectrum, it doesn't have enough statistics for these protons. As noticed previously, good statistics requires a large value of source particles. The choice for this value was made as a balance between the reasonable wait time of a FLUKA's run and the number of the source history particles used in the paper's experiment. The Aghara paper reports a minimum of 20 million history particles [2]. Given the fact that this experiment required at least 10 runs to get small uncertainty in the FLUKA's output, the minimum number of history particles used in the paper experiment was already close to the highest value, which would make the wait time of the FLUKA's run unreasonably long.

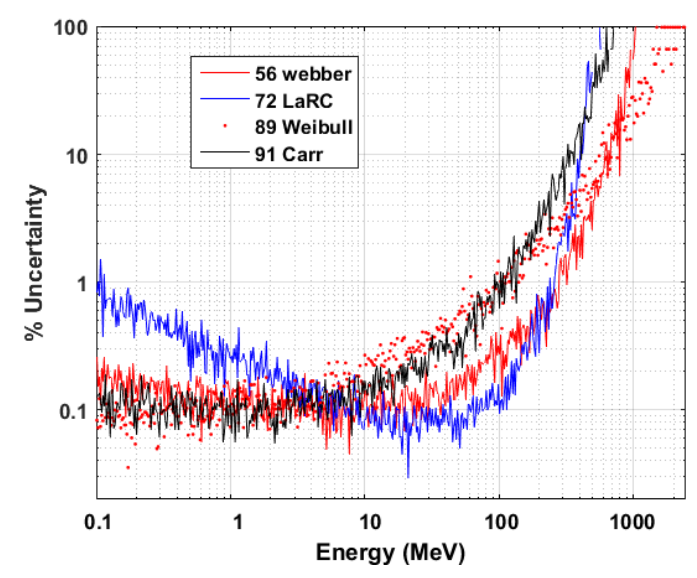

Figure 10 Percent uncertainty in the USRBDX result

Therefore, the same 20 million source history particles were set in the FLUKA's input. As a result, the output was obtained with a good data density and at the same time with uncertainties well below $1 \%$ for most of the energy spectra. One exception is an abnormal high uncertainty of the $72 \mathrm{LaRC}$ for protons with low energy. 
The output of the USRBDX card has units of $\mathrm{part} / \mathrm{cm}^{2} / \mathrm{GeV} / \mathrm{pr}$. The fluence in Figure 9, however is given in $\mathrm{part} / \mathrm{cm}^{2} / \mathrm{MeV}$. The following is the conversion procedure:

$$
\left[\frac{p a r t}{\left(\mathrm{~cm}^{2}\right)(\mathrm{GeV})(\mathrm{pr})}\right]\left[\frac{1 \mathrm{GeV}}{10^{3} \mathrm{MeV}}\right]\left[\mathrm{A}_{\mathrm{d}} \mathrm{cm}^{2}\right]\left[N \frac{\mathrm{pr}}{\mathrm{cm}^{2}}\right]
$$

where $A_{d}$ is the detector's area normal to flux and the fourth term is the normalization factor. Table 9 shows normalization parameters calculated for all four sources using eq.10. The detector's area is $\pi \cdot 15^{2}=706.86 \mathrm{~cm}^{2}$, were 15 is the detector's radius.

Table 9 Normalization factors for SPE source

\begin{tabular}{|c|c|}
\hline SPE Event & Normalization factor $\left(\mathbf{p r} / \mathbf{c m}^{2}\right)$ \\
\hline 56 Webber & $9.5048 \mathrm{e}+09$ \\
\hline 72 LaRC & $1.8439 \mathrm{e}+10$ \\
\hline 89 Weibull & $4.0692 \mathrm{e}+11$ \\
\hline 91 Carr & $1.0337 \mathrm{e}+12$ \\
\hline
\end{tabular}

The second goal was achieved by employing the USRBIN scoring card. Unlike the USRBDX, which scores per area, the USRBIN card scores a 3-D region. The user is required to choose a grid (mesh) type that partitions the scoring region (i.e. water slab in case of goal 2) into elements or grid bins.

There are several options for the grid's type. For a Cartesian grid, the user specifies the dimensions of a rectangular parallelepiped that encompasses a scoring region. Then, the user chooses the number of bins that fit along each dimension of that parallelepiped. Another type of the grid is cylindrical. In this case, the scoring region is a right circular cylinder. In the Aghara paper, the dose distribution was scored only within the water part of the target which is cylindrical. Therefore, the natural choice is cylindrical grid. The thickness of the water slab is $30 \mathrm{~cm}$ and 300 bins were chosen.

The default output of USRBIN is GeV/g/pr. To obtain the dose per primary particle, Gy/pr, the following conversion is necessary:

$$
\left[\frac{\mathrm{GeV}}{(g)(p r)}\right]\left[\frac{10^{9} \mathrm{eV}}{\mathrm{GeV}}\right]\left[\frac{1.60218 \times 10^{-19} \mathrm{~J}}{1 e V}\right]\left[\frac{10^{3} g}{\mathrm{~kg}}\right]=\frac{\mathrm{Gy}}{p r}
$$

Next, a normalization factor converts it into a dose as follows: 
where the second term is the same normalization factor as in goal 1. The third term is a cross-sectional area of the target perpendicular to flux. The result of the goal 2 is shown in figure 11 . The first thing to notice here is how close the FLUKA and OLTARIS outputs are. This is unexpected result since unlike FLUKA, OLTARIS is deterministic, not an MC code. The second thing to notice is very small uncertainties for 56 Webber and 72 LaRC spectras while uncertainties for 89 Weibull and 91 Carrington are noticeably larger.
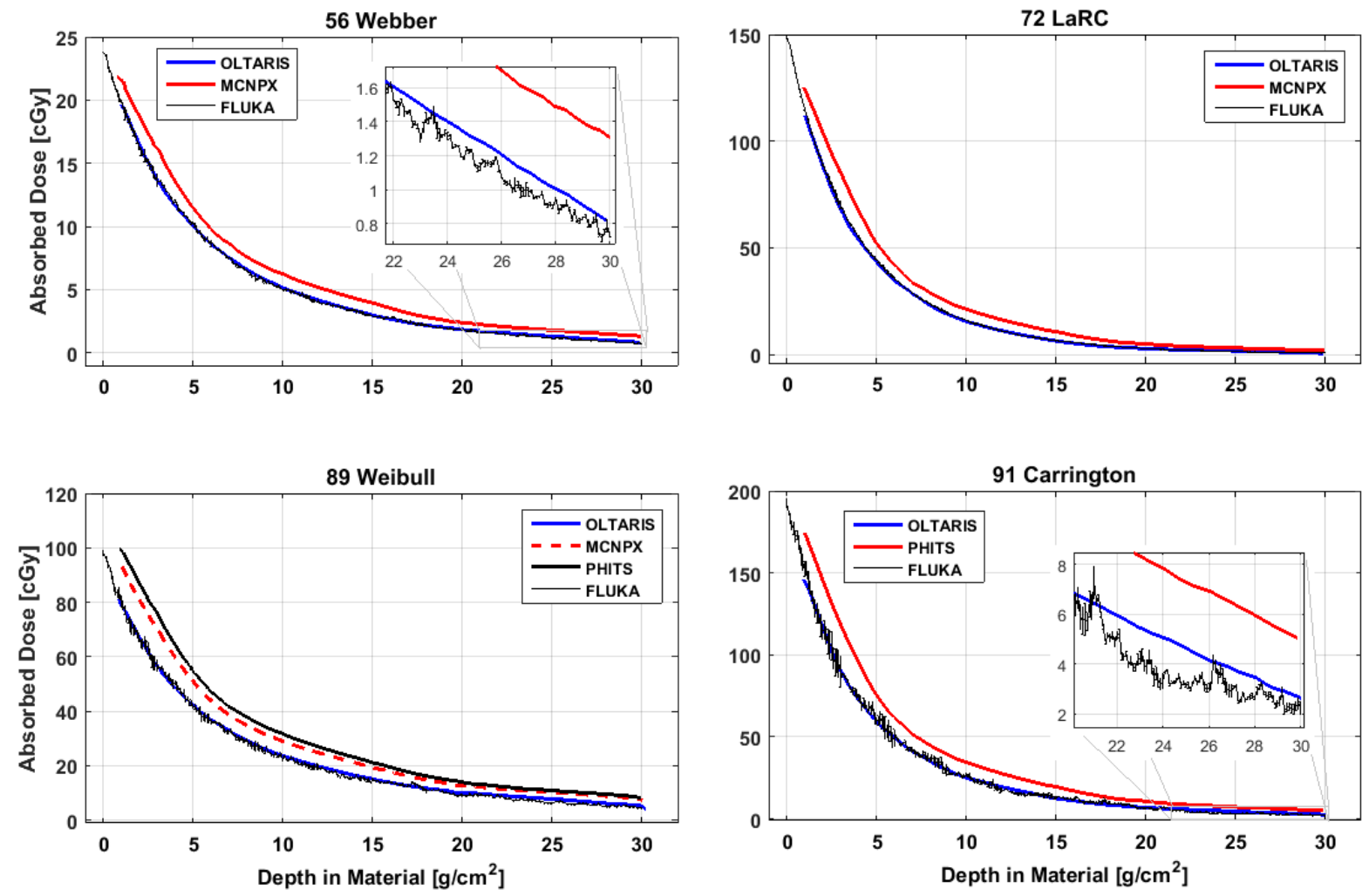

Figure 11 Dose distribution within the water slab shielded by $10 \mathrm{~g} / \mathrm{cm}^{2}$ of aluminum shield for: (a) 56 Webber, (b) 72 LaRC, (c) 89 Weibull and (d) 91 Carrington

Table 10 shows the MSD numbers (eq.14) normalized by the highest dose. These numbers allow to make a quantified comparison of the results obtained by four different transport codes. Notice how MCNPX and PHITS have the same numbers except in the case of 89 Weibull source. This is the only case where PHITS can be distinguished from the MCNPX. In fact, in this case, the MSD for PHITS is about twice as large as for MCNPX because the difference between the FLUKA's and MCNPX results is smaller than 
between FLUKA and PHITS (fig 11). The fact that FLUKA is very close to OLTARIS is manifested in much smaller MSD numbers when compared to MCNPX.

Table 10 MSD Values for Doses in figure 11 to compare FLUKA, MCNPX and OLTARIS results

\begin{tabular}{|c|c|c|c|}
\hline & $\begin{array}{c}\text { FLUKA Result } \\
\text { vs MCNPX }\end{array}$ & $\begin{array}{c}\text { FLUKA Result } \\
\text { vs PHITS }\end{array}$ & $\begin{array}{c}\text { FLUKA Result } \\
\text { vs OLTARIS }\end{array}$ \\
\hline 56 Webber & 0.0594 & 0.0594 & 0.0007 \\
\hline 72 LaRC & 0.3081 & 0.3081 & 0.0041 \\
\hline 89 Weibull & 0.5145 & 1.0254 & 0.0097 \\
\hline 91 Carrington & 0.8184 & 0.8184 & 0.0157 \\
\hline
\end{tabular}

A possible explanation why the FLUKA output is closer to the analytic OLTARIS rather than to MC MCNPX is the nature of the simulation. The set-up of the target model and detector were essentially onedimensional because the dose was scored along the axis of the target averaging the lateral variations. The MCNPX, on the other hand, might have a different detector configuration thus yielding slightly different result.

As mentioned earlier, the reason of different uncertainties between top two cases in figure 11 is most likely related to the mechanism FLUKA uses to generate the source fluence.

\subsection{GCR Validation}

The same validations were performed with GCR being a source. Unlike the Aghara simulation, the GCR source in Fluka is defined as an emission sphere instead of a pencil beam. Consequently, detectors and targets are also spheres instead of cylinders. The target for goal 1 is just a vacuum sphere with radius of $10 \mathrm{~cm}$ inside a vacuum void. The radius of the emission sphere is $80 \mathrm{~cm} .5 \times 10^{7}$ events were simulated and repeated for 15 runs. The result is shown in figure 12 where part $a$ is a figure adopted from the Fluka Cosmic Rays Course [18] which shows fluence of four types of particles: protons, $\alpha$-particles, carbon and iron ions. Part $b$ shows how well the FLUKA's output matched part $a$. Notice the increase in uncertainties for heavy ions. The reason is similar to that explained for the SPE spectra: heavy ions are much less abundant in the GCR flux than light protons, thus the statistical error is larger [으. In addition, figure $12 b$ contains an insert - a zoomed-in region of the $\alpha$-particles showing the scale of uncertainty of the light particles while uncertainties of two heavy particles spectras are noticeably larger. 


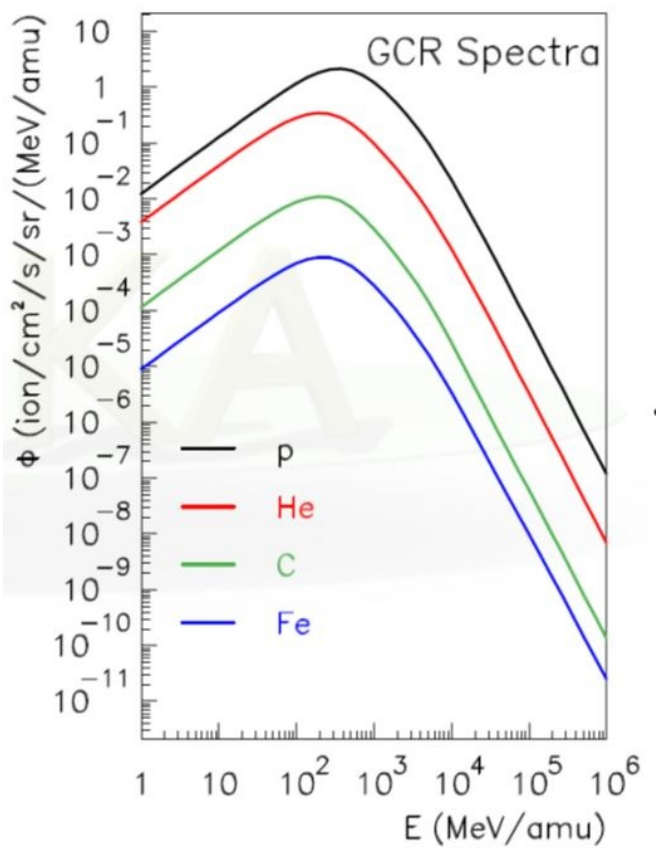

(a)

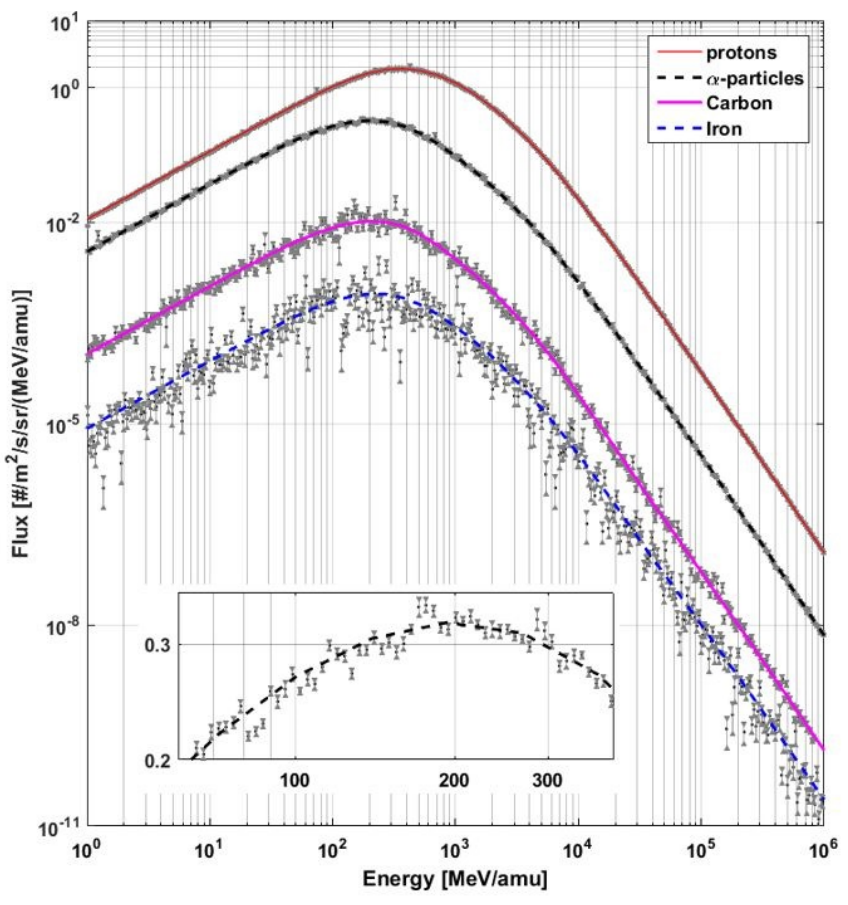

(b)

Figure 12 (a) FLUKA's GCR source proton spectrum, $\alpha$-particles, carbon and iron ions, (b) FLUKA's output of the flux scoring detector

The fluence in figure 12 is given in ions $/ \mathrm{cm}^{2} / \mathrm{s} / \mathrm{sr} /(\mathrm{MeV} / \mathrm{amu})$, where amu is an atomic mass number of a given ion. The conversion from the USRBDX units (integral over solid angle) is as follows:

$$
\left[\frac{\text { part }}{\left(\mathrm{cm}^{2}\right)(\mathrm{GeV})(\mathrm{pr})}\right]\left[\frac{\mathrm{GeV}}{10^{3} \mathrm{MeV}}\right]\left[\frac{1}{1 / \mathrm{amu}}\right]\left[\frac{10^{4} \mathrm{~cm}^{2}}{\mathrm{~m}^{2}}\right]\left[\frac{1}{2 \pi \mathrm{sr}}\right]\left[\frac{\mathrm{pr}}{\mathrm{s}}\right]=\frac{\text { ions }}{\left(\mathrm{m}^{2}\right)(\mathrm{s})(\mathrm{sr}) \frac{\mathrm{MeV}}{\mathrm{amu}}}
$$

where $s r$ stands for steradians. The last term is the normalization factor found, as explained earlier, by dividing the Fluxst by the Flux value found in the *.out file.

$\underline{\text { Goal } 2}$ for the GCR source was performed using data from the Radiation Assessment Detector (RAD) mentioned earlier. This radiation detector on board of the Mars Science Laboratory measured radiation background during transit to Mars. During the cruise period, the solar activity was low to moderate. The GCR dose rate in the RAD’s silicon detector was measured to be $332 \pm 23 \mu \mathrm{Gy} /$ day [26]. Notice the units are in dose, not in equivalent dose. Two crude models of the RAD instrument located inside the MSL transit module were constructed in Fluka. One is a silicon disk inside an aluminum spherical shell (figure 13), another is the same disk but with no shield. 


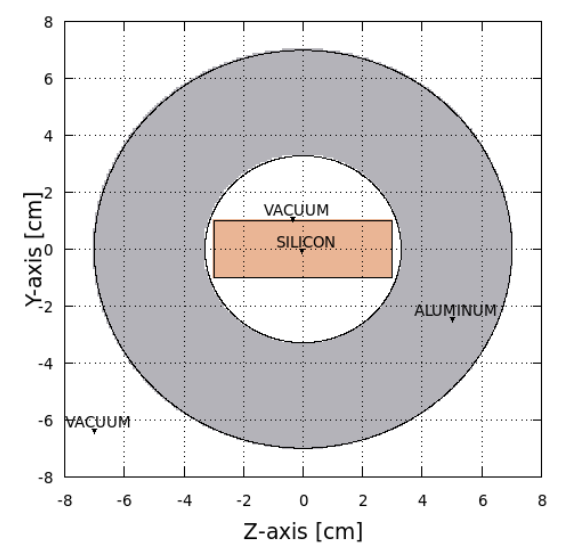

Figure 13 Fluka model of $R A D$

The shield thickness is $10 \mathrm{~g} / \mathrm{cm}^{2}$ because that was the thickness of the actual shield around most of the solid angle around the RAD instrument. Unlike the Aghara paper where the goal was to obtain dose distribution along the thickness of a target, this experiment requires just one value to compare with the measurement obtained by RAD. Consequently, the USRBIN card was set a bit differently. Instead of using mesh that divides the target into a number of bins, the scoring was done per region meaning a dose absorbed by a target as a whole was scored producing a single value as a result. Since the scoring is done per region, the output unit of the USRBIN card differ from the one used in Aghara experiment. The bins of the Cartesian and cylindrical grid have simple shapes and their volume is calculated analytically. When the scoring is done per region, the bins can be of any shape because regions can be described in any unpredictable ways. Consequently, unlike the case when the scoring is done with Cartesian or cylindrical grid, the output of the scoring per region is not normalized to the region volume. The output units of the USRBIN card in this case is the same unit as before but multiplied by region's volume [8]. The unit conversion to the dose rate is as follows:

$$
\left[\frac{(\mathrm{GeV})\left(\mathrm{cm}^{3}\right)}{(\mathrm{g})(\mathrm{pr})}\right]\left[\frac{1}{\mathrm{~cm}^{3}}\right]\left[\frac{10^{9} \mathrm{eV}}{\mathrm{GeV}}\right]\left[\frac{1.60218 \times 10^{-19} \mathrm{~J}}{1 \mathrm{eV}}\right]\left[\frac{10^{3} \mathrm{~g}}{\mathrm{~kg}}\right]\left[\frac{\mathrm{pr}}{\mathrm{s}}\right] \cdot\left[10^{6} \cdot 3600 \cdot 24\right]=\frac{\mu G y}{d a y},
$$

where the second term is divided by the region's volume, the $6^{\text {th }}$ term is Fluxst/Flux and the last term is conversion to $\mu G y$ and days. The FLUKA's output for the case with shielding is $2.011 \mathrm{e}-6 \mathrm{GeV} \cdot \mathrm{cm}^{3} / \mathrm{g} / \mathrm{pr} \pm$ 14.6\%. After conversion to dose rate this becomes $289.34 \pm 42 \mathrm{uGy} /$ day. For the case without shielding: $1.5624 \mathrm{e}-6 \pm 40.8 \%$ and $224.8 \pm 92 \mathrm{uGy} /$ day respectively. Figure 14 gives a comparative perspective of these 
two results together with the RAD’s result. The reason why the unshielded silicon detector absorbs less dose in a given time than the shielded one is probably due to the absence of shielding that eliminates secondary radiation which, in turn, increases radiation behind a shield. The uncertainty overlap between the RAD's measurement and the FLUKA's output for the shielded model is larger than for unshielded one. In any case, this experiment validates that even a crude model of radiation detector yields output that is within uncertainty limits of the actual measurement performed in the interplanetary space. This validates the FLUKA's GCR model.

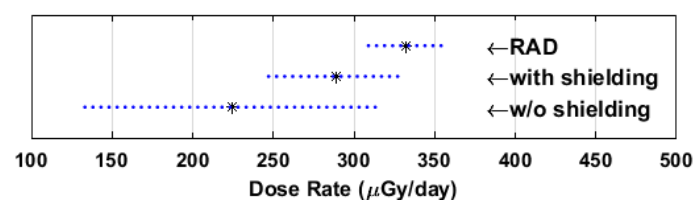

Figure 14 Overlap of the RAD measurements with fluka simulation output 


\section{EXPERIMENTS}

Once FLUKA's output is validated, it is possible to start testing materials for their shielding properties. The first step is to set up a testing configuration that includes a target, a shield around it and a source (SPE and GCR).

\subsection{Setting up a New Testing Configuration}

The dose absorbed by a target depends on the configuration of target and source. The intention of this study was to set up a 3-D model that represents the real world as close as possible. The experiment in the Aghara's paper is not a good example. In that paper the type of source (beam) and the target (cylinder) are rather 1-D models because dose is scored axially along the cylinder while the radial variations are not considered and are averaged. The Aghara's experiment has two other simplifications. First, the water slab follows the shield immediately while in reality the target (human) is floating in certain volume (usually a cylinder) filled with air which, in turn, is surrounded by a shield. The second simplification is that human tissue was represented by water. This might have an effect too. Also, since secondary radiation affects absorbed dose and because it depends on the thickness of a shield, the scale of selected model might have some effect on the dose absorbed by a target - this paper's ultimate quantity of interest. To summarize, the following must be modified or added to the Aghara's set-up to get a more realistic model:

- $\quad$ source as a flux, not a 1-D beam

- $\quad$ tissue instead of water

- $\quad$ air buffer between target and shield

- $\quad$ 1:1 scale of a shield

\subsubsection{The Source}

The source was modeled as a uniform and isotropic SPE fluence with energy spectrum calculated by the Band function (eq.8-9). This is similar to the GCR case where particles are generated on the emission surface. In the first case, however, source is a built-in tool and therefore the normalization factor is calculated automatically. In case of SPE with spherical source, a normalization factor is found manually with additional correction factor which accounts for the fact that particles are generated on the surface of a sphere rather than from a single point. This factor was found empirically by scoring the source fluence generated by the Band 
function and comparing it with corresponding fluence reported by a published paper. It was found that this factor is proportional to the area of emission sphere and inversely proportional to the radius of detector:

$$
A_{s}\left(\frac{1}{5 r_{d}}\right)^{2} \frac{\mathrm{cm}^{2}}{\mathrm{~cm}^{2}}
$$

where $r_{d}$ is the detector's radius. Equations (14) and (16) are multiplied by this factor. The detector's area, which appears in both equations, cancels with $r_{d}$ in the correction factor and simplifies to:

$$
\left(\frac{4}{5} \pi \cdot r_{s}\right)^{2} \frac{\mathrm{cm}^{2}}{\mathrm{~cm}^{2}}
$$

The conversion steps from the raw FLUKA's result to fluence and to absorbed dose in case of isotropic source therefore becomes:

USRBDX

$$
\left[\frac{p a r t}{\left(\mathrm{~cm}^{2}\right)(\mathrm{GeV})(\mathrm{pr})}\right]\left[\frac{1 \mathrm{GeV}}{10^{3} \mathrm{MeV}}\right]\left[N \frac{\mathrm{pr}}{\mathrm{cm}^{2}}\right]\left(\frac{4}{5} \pi \cdot r_{\mathrm{s}}\right)^{2} \frac{\mathrm{cm}^{2}}{\mathrm{~cm}^{2}}
$$

USRBIN

$$
\left[\frac{\mathrm{GeV}}{(\mathrm{g})(\mathrm{pr})}\right]\left[\frac{10^{9} \mathrm{eV}}{\mathrm{GeV}}\right]\left[\frac{1.60218 \times 10^{-19} \mathrm{~J}}{1 \mathrm{eV}}\right]\left[\frac{10^{3} \mathrm{~g}}{\mathrm{~kg}}\right]\left[N \frac{\mathrm{pr}}{\mathrm{cm}^{2}}\right]\left(\frac{4}{5} \pi \cdot r_{\mathrm{s}}\right)^{2} \frac{\mathrm{cm}^{2}}{\mathrm{~cm}^{2}} \cdot 100=c G y
$$

\subsubsection{The Target}

An ICRU tissue equivalent representing the chest of a patient was used as a target in this study. Table 11 lists the elemental compositions of the simulated ICRU tissue [16]. This tissue material was validated by measuring the inelastic scattering length in ICRU sample of certain thickness due to the beam of seven different ions with different energies.

Table 11 ICRU Elemental composition (wt.\%) of soft tissues in the human body used in FLUKA simulations [16]

\begin{tabular}{|c|c|c|c|c|}
\hline & $\mathrm{H}$ & $\mathrm{C}$ & $\mathrm{N}$ & $\mathrm{O}$ \\
\hline ICRU & 10.1 & 11.1 & 2.6 & 76.2 \\
\hline
\end{tabular}

The Porta paper reports a result that may be compared to the Fluka output can be compared with paper's result. Table 12 is adopted from the Porta's paper and shows inelastic scattering length found there. A column was added that shows the corresponding inelastic lengths found with Fluka. All but alpha particle show a good match. 
Table 12 Inelastic Scattering Length Into ICRU Tissue as a Function of Ion and Energy [16]

\begin{tabular}{|c|c|c|c|c|}
\hline \multirow{2}{*}{ Ion Beam } & Charge State & Energy & Inelastic scattering length & FLUKA \\
\cline { 3 - 5 } & & $(\mathrm{MeV} / \mathrm{u})$ & $(\mathrm{cm})$ & $(\mathrm{cm})$ \\
\hline $\mathrm{H}$ & +1 & 200 & 107.0 & 107.0 \\
\hline $\mathrm{He}$ & +2 & 202 & 50.55 & 28.51 \\
\hline $7 \mathrm{Li}$ & +3 & 234.3 & 30.18 & 29.68 \\
\hline $\mathrm{B}$ & +5 & 329.5 & 23.38 & 23.24 \\
\hline $\mathrm{C}$ & +6 & 390.7 & 22.14 & 22.10 \\
\hline $\mathrm{N}$ & +7 & 430.5 & 20.41 & 20.34 \\
\hline $\mathrm{O}$ & +8 & 468 & 18.88 & 18.83 \\
\hline
\end{tabular}

The first attempt to model a spacecraft with a person inside was as follows. The spacecraft was modeled as a cylinder of various material with radius of $400 \mathrm{~cm}$ and length of 800: about the dimensions of a typical ISS module. The thickness was varied from 10 to $100 \mathrm{~g} / \mathrm{cm}^{2}$. The correction factor discussed earlier (eq.19), however, was empirically found only for spherical detector so tests of cylindrical targets inside spherical source were not possible. Therefore a spherical spacecraft was modeled instead. This model consisted of a spherical aluminum shell with four thicknesses: 10, 20, 30 and $50 \mathrm{~g} / \mathrm{cm}^{2}$ and with fixed inner radii of $400 \mathrm{~cm}$ (fig 15a). The target is a sphere with radius of $30 \mathrm{~cm}$ made of human tissue defined above.

However, there are two problems with the 1:1 scaling in FLUKA. First, FLUKA's run slows down significantly when large objects are used. Each experiment performed for this study required numerous repeated runs and it would be impractical to perform all of them using real scale models. The second problem is a large uncertainty in the target dose when the target size is much smaller than a shield.

These issues were addressed by the elimination of an air "gap" such that the target and the shield are in direct contact (fig 15b). To understand how this modification affects the results, a test was performed on both “realistic” and simplified configurations. 


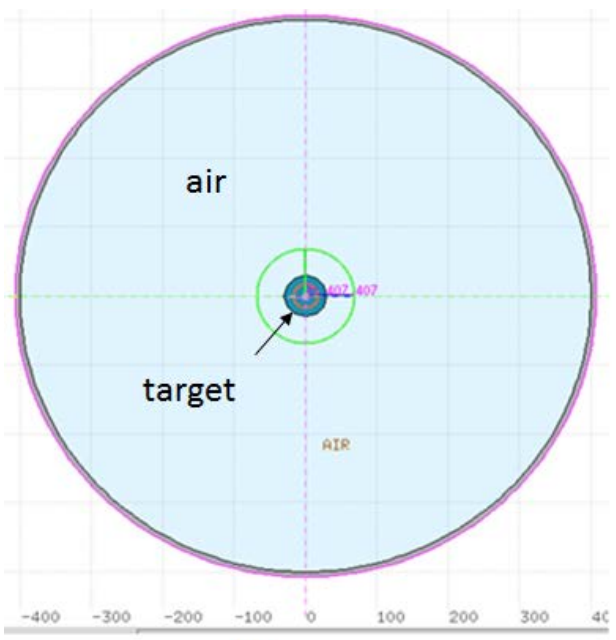

(a)

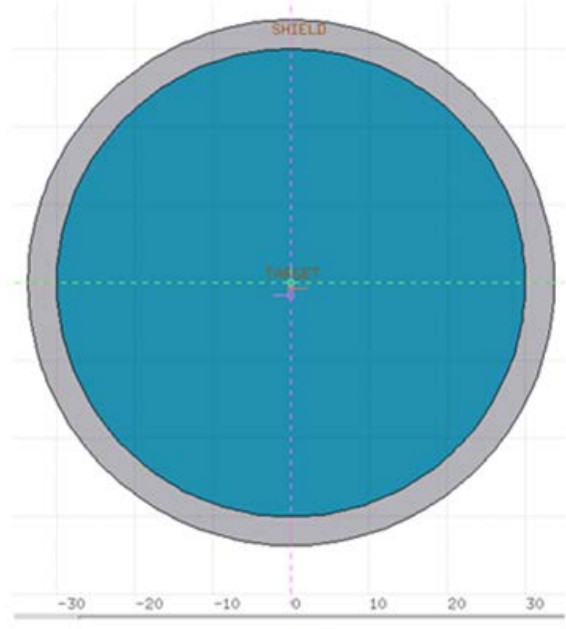

(b)

Figure 15 Two Models of a Spacecraft with a "human" target inside. (a) - realistic. (b) - simplified

Four SPE events were tested: Feb 23, 1956; Apr 29 1973; Oct 1989 and Jan 20, 2005. The dose absorbed by the target was recorded for four shield thicknesses using two models and then compared. The results show different degree of agreement between realistic and simplified configurations (figure 16). The 1973 event causes the least and the 2005 the largest difference. In case of 1956 and 2005 events the simplified configuration values are outside the FLUKA's uncertainties of the realistic model and underestimate the realistic values. In case of 1973 and 1989, however, it is possible to say that simplified values are within the uncertainties of realistic configuration and it is safe to assume that simplified configuration yield the same absorbed dose results as the realistic one. 

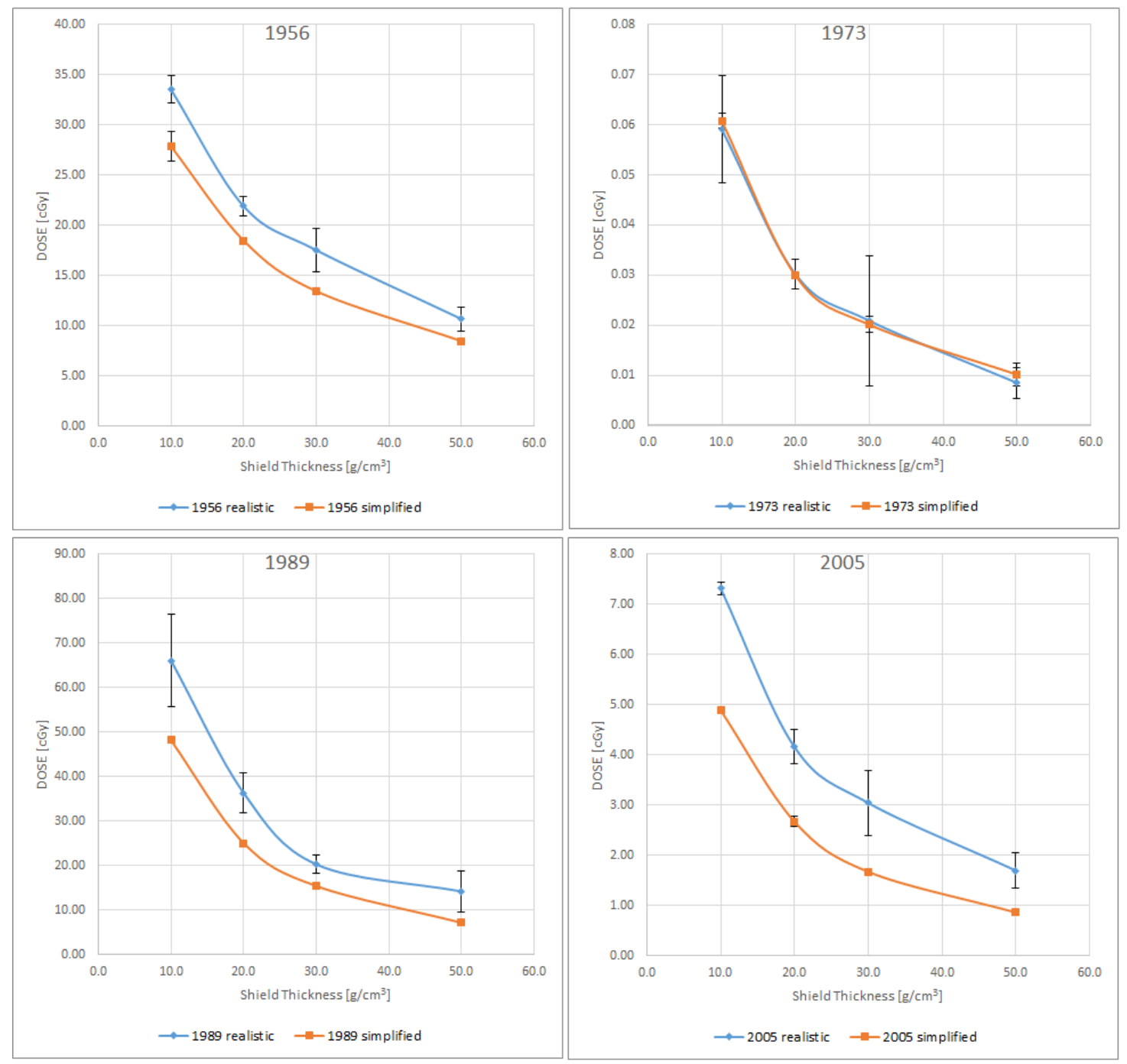

Figure 16 Difference in Dose Absorbed by the Target Between "Realistic" and "Simplified" Models for Four SPE Events

\subsection{Effect of the Distance Between Souce and Target on the Resultant Absorbed Dose}

Since the correction factor for the isotropic source was found empirically, it is important to make sure that the distance between the source and the target has no effect on the resultant dose absorbed. A series of tests were performed on the simplified model with the aluminum shield of $10 \mathrm{~g} / \mathrm{cm} 2$ thick. The radius of the spherical source was varied such that the distance from the outer surface of a shield to the source increased from 20 to $120 \mathrm{~cm}$. The 1989 SPE event was chosen as the source. Figure 17 shows results for the dose absorbed by both shield and target. It can be seen that almost all data points are within the smallest uncertainty. The only exeption are 20 and $120 \mathrm{~cm}$ for the shield. This means that as long as the source is in between this 
range, the resultant dose is not affected by how far away the source is. Therefore it was decided that it is safe to assume that the empirical correction factor does account for the source radius as long as it is within the aforementioned range. Anyway, to eliminate any possible effect of the source's proximity to the outcome of an experiment, all subsequent tests were performed with the fixed source distance of $46 \mathrm{~cm}$.

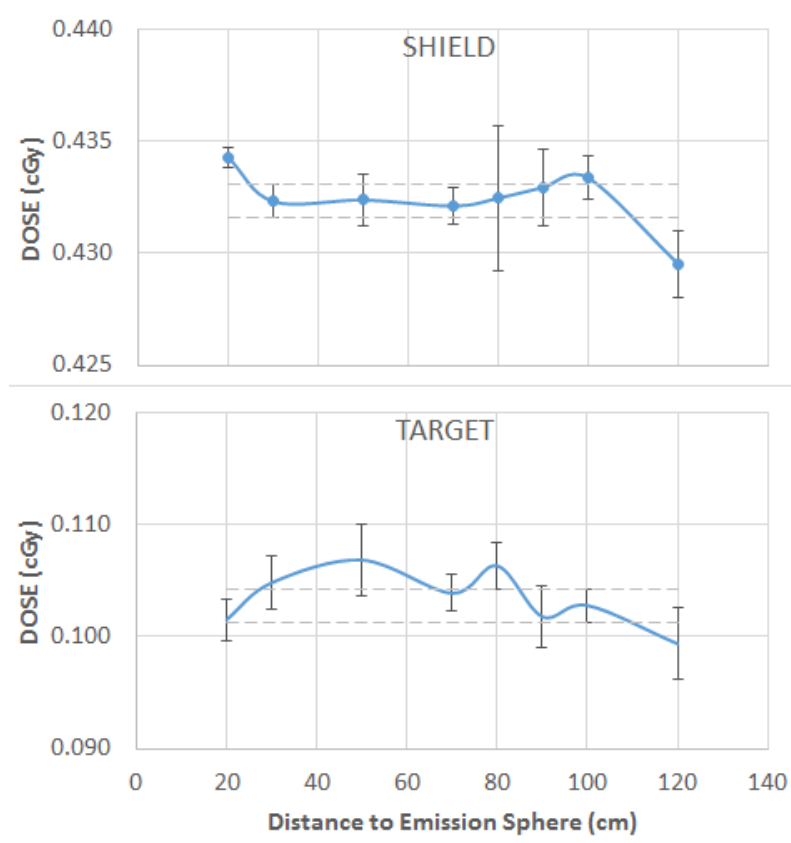

Figure 17 Influence of distance between source and target on dose absorbed

\subsection{Multylayer Approach and Target Set-Up}

The usual way to demonstrate the shielding property of certain material is to show the dose profile along the thickness of the shield made of this material. In this study, such a profile was obtained in the following way. The shield region of the simplified model was divided into twelve layers (fig 18). The dose absorbed by each layer was scored with USRBIN per region card so that the profile of twelve values can be constructed. In addition to the dose distribution within shield, dose was also scored for the target as a single value in order to see what overall effect different shielding materials make on the target (human tissue).

This approach was validated in the following way. The cylindrical water part of Aghara'a paper model was divided into thirty sections with fixed length. The dose absorbed by each section was scored with USRBIN per region card. Then, the resultant thirty dose values were superimposed on figure 11. The 1956 Webber source was used (eq. 11). The result is shown in figure 18, which also has an insert with the region of the worst 
agreement. "Mesh" in the figure implies the curve from figure 11 which is the dose profile obtained with the cylindrical grid of 500 bins along the axis of the water cylinder (see mesh explained earlier). In other words, "mesh" is the same curve as in figure 11 while "per region" is the curve that connects those thirty dose values explained above. The resultant "per region” values look close to the "mesh" curve. The difference between them in terms of normalized MSD is 0.0036 . This is about five times bigger (worse agreement) than the MSD number for FLUKA vs OLTARIS but almost 85 times smaller (better agreement) than the MSD for FLUKA vs MCNPX (see table 10).

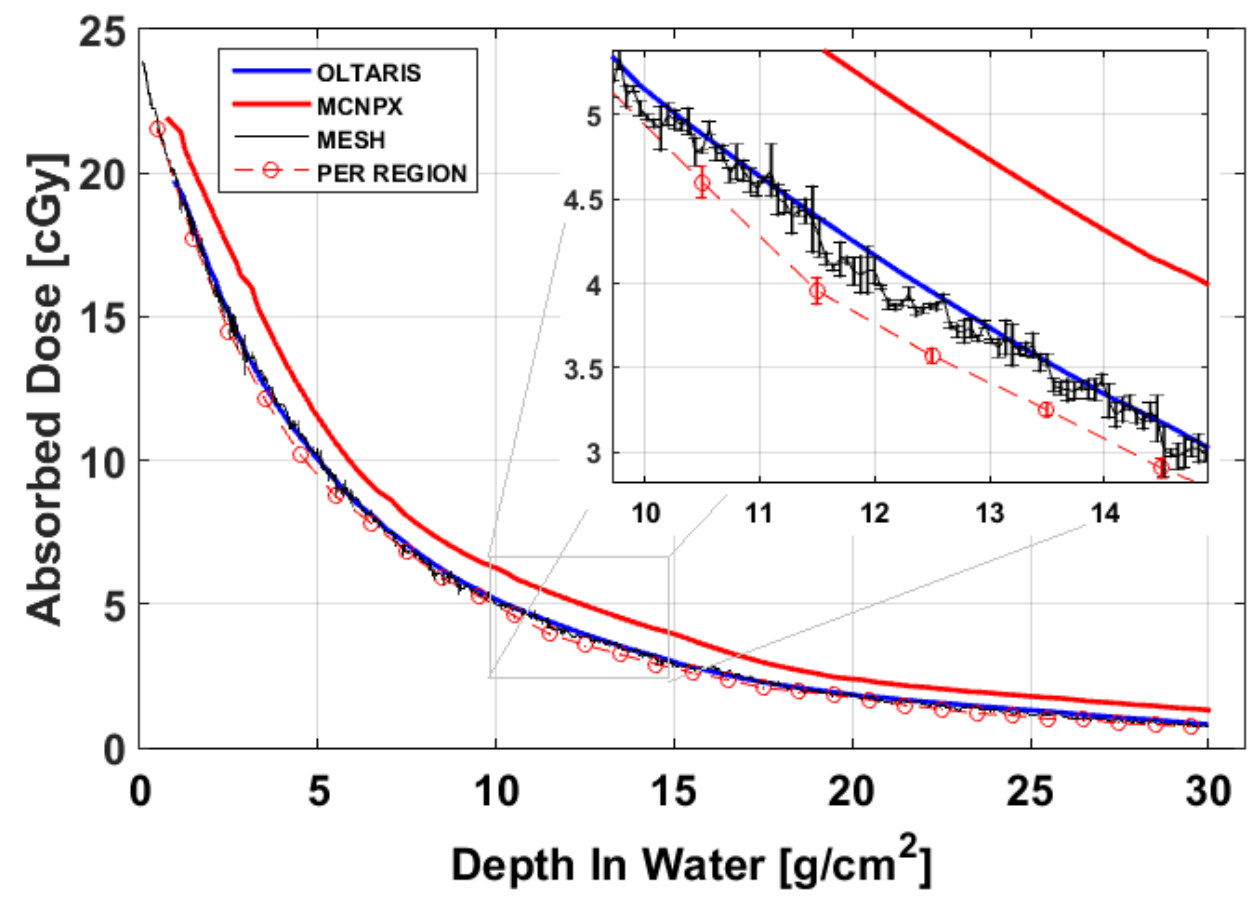

Figure 18 Validation of Multilayer Approach to Find Dose Within Shield (source: 1956 Webber)

Another way to validate this approach is, once again, to compare results from Fluka to those from a similar experiment reported in a published paper [17]. In that paper the material thicknesses were of 1, 5, 10, 20, 30, 50, and $100 \mathrm{~g} / \mathrm{cm}^{2}$ and a smooth line through these points was displayed. The source used in the paper is Band model for the series of SPEs that occurred during the 19-24 October 1989 time period. Figure 19figure shows the differential fluence of the source used in the Rojdev paper with fluence obtained by eq-s 8 and 9 as well as corresponding Fluka output superimposed. The Band model parameters for these equations were obtained by averaging parameters of four events occurred in October 1989 (Table 4). 


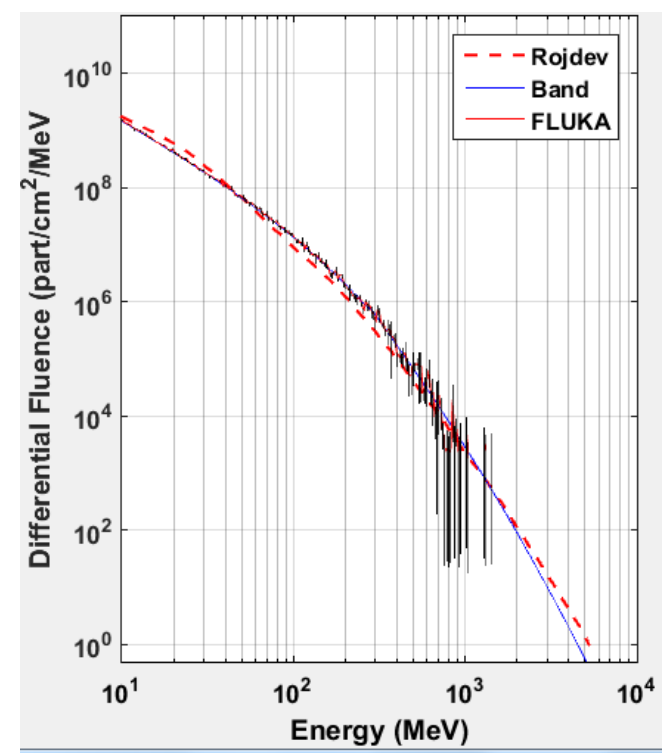

Figure 19 Comparison of the Oct 1989 Band Fits:

Rojdev, Theoretical and FLUKA

It is not clear whether the Rojdev paper used the same method because, as figure 19 reveals, there is slight deviation in the fluence spectra between the aforementioned method and Rojdev's method. Table 13 shows the MSD comparison values. Obviously, the fluences obtained by FLUKA and from the Band equation have the best agreement since one is generated from the other. The agreement between the Band equation and the flux reported by Rojdev is much worse but still has the same magnitude of MSD as the 89 Weibull fluence from figure 9 .

Table 13 Comparison between fluences obtained by FLUKA, Rojdev and Band

\begin{tabular}{|c|c|}
\hline & MSD Values $^{*}$ \\
\hline FLUKA vs Rojdev & 153.00 \\
\hline FLUKA vs Band & 6.88 \\
\hline Rojdev vs Band & 161.76 \\
\hline
\end{tabular}

* - \#/1E5

Figure 20 shows four important comparisons:

a. the difference in dose profile between two configurations:

- beam source with a cylindrical target as in the Aghara's paper

- flux source with the multilayer shperiacal shield configurations used farther on in this study.

b. the dose profile within an aluminum shield due to the Band fitted 1989 source: 
- $\quad$ adopted from the Rojdev paper vs

- corresponding Fluka output from multilayer configurations

Both comparisons validate the multilayer approach. Comparison (b) has slightly worse fit between Rojdev's and FLUKA's profiles than the same fit in the comparison (a). It is unclear whether this is a consequence of a source misalignment (fig. 20) or if it is due to differences between the MC and analytic transport codes. The Rojdev paper used the 2010 version of HZETRN [17]. The comparison (a) sahows almost perfect agreement because the same source and transport code were used and it provides the best validation that multilayer approach yields the same results as a single layer, mesh USRBIN scoring method. This is convincing evidence that the multilayer method explained above is sufficient for the shielding studies. However, noticeable differences between comparisons (a) and (b) motivates more realistic modeling.

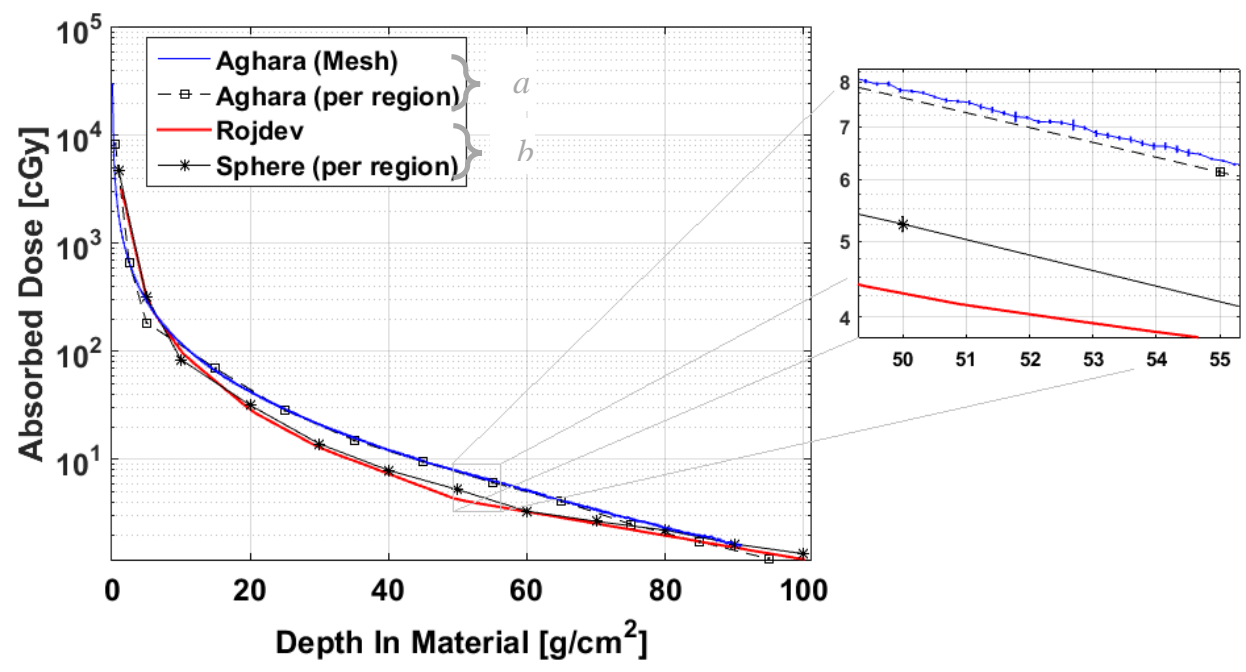

Figure 20 Comparison in Dose Profile for Aluminum Shield Between FLUKA and Rojdev Paper Results. Comparison Between Aghara and Spherical target Set-Ups (source: 1989 Band Fit)

\subsection{Choosing the Solar Event to Use as a Source}

Once the material evaluation method was defined and validated, a solar event for all farther experiments should be chosen. The obvious choice of a source SPE for material testing would be the largest one. Figure 21 depicts eight largest GLEs obtained with Band fitting method using parameters listed in the Tylka paper [22] The GLE occurred in 2003 seems to have the highest fluence of low energy protons but 1956 event has highest fluence of protons with energy of $300 \mathrm{MeV}$ and higher. It is not clear what part of the spectrum contributes 
most to the overall dose absorbed by shield and target. In other words, what event has highest influence on the target.

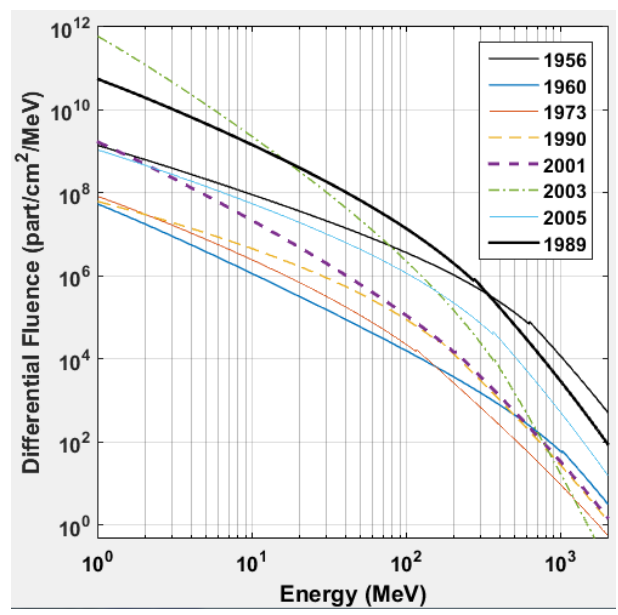

Figure 21 Proton Fluence of the Largest SPEs (Band Fit)

To find it out, a series of tests were performed where the simplified configuration with the shield divided into twelve layers was exposed to eight SPEs. Figure 22 shows results for the dose profiles in the shield were part $a$ depicts two events which has the most influence on the target's dose. Figure 23 shows how much dose the target absorbs due to each SPE. The 2003 event is presented in both parts for comparison purposes to demonstrate how the SPEs with small influence are compared to those with large influence. This figure makes it clear that 1956 and 1989 events are the most influential and will be used in the subsequent experiments.

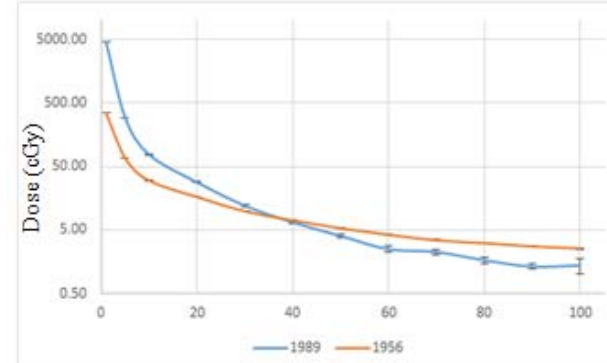

(a)

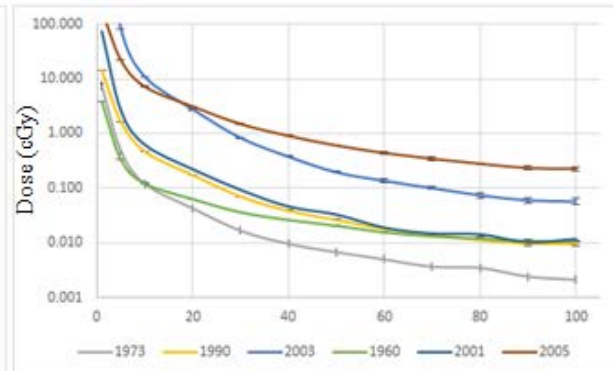

(b)

Figure 22 Dose Profile Within $100 \mathrm{~g} / \mathrm{cm}^{2}$ Thick Aluminum Shield Due to 8 Largest SPEs: (a) Most Influential, (b) Least Influential 


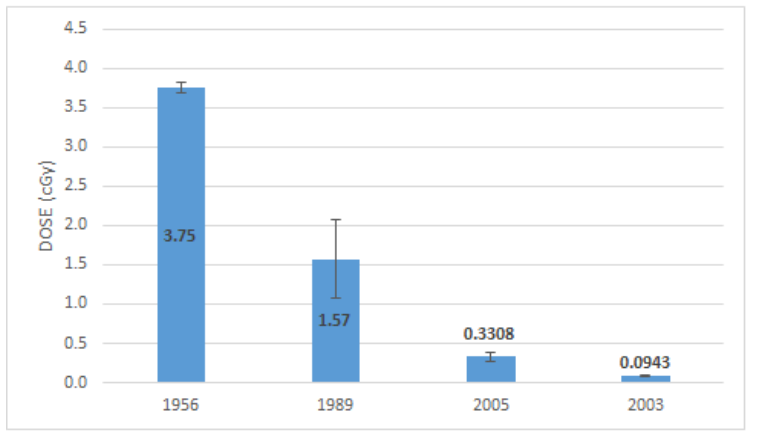

(a)

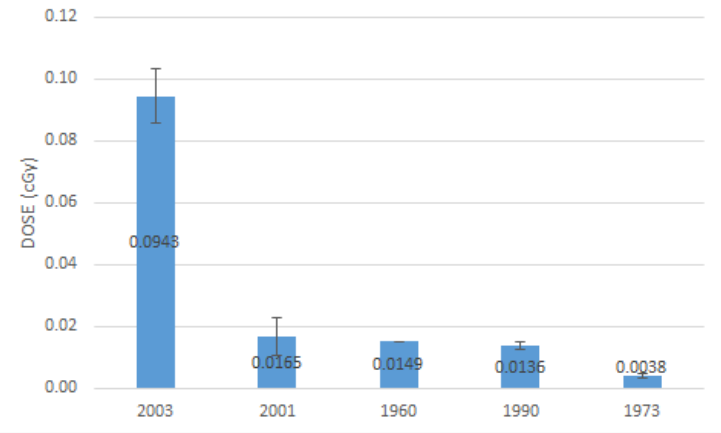

(b)

Figure 23 Dose Absorbed by the Target Due to 9 Largest SPEs. (a) Most Influential, (b) Least Influential

\subsection{Dose Profiles Within Diferent Materials and Resultant Target Doses}

This experiment was performed to determine effectiveness of different materials to shield from SPEs, specifically the Band model for 1956 and 1989 events. Fourteen elements from the periodic table as well as polyethylene and water were tested. The simplified model depicted in Figure 24 was used. A single material was assigned to all twelve layers of the shield. As explained earlier, the dose absorbed by each layer was scored to get a dose distribution within the shield.

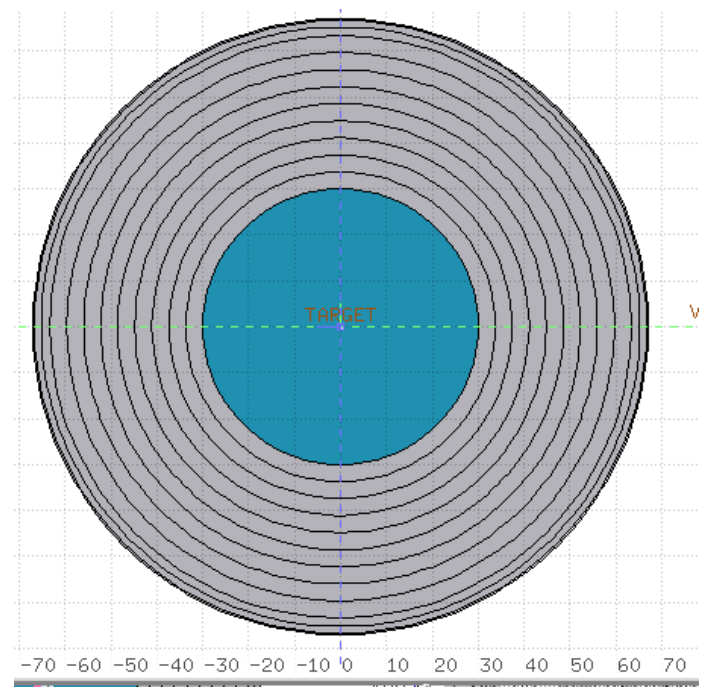

Figure 24 The Simplified Model to Obtain the Dose Profile Within the Shield

Figure 25 shows the dose profile for all test materials. The first thing to notice here is how close are the dose distributions for all elements. Since aluminum is the dominant component in most of the modern spacecraft's structure, this metal was served as a reference for the rest of the test materials. For that reason it 
is bolded in the plot. Aluminum shows better performance than both polyethylene and water. Both are hydrogen-rich and, as explained previously, hydrogen has the highest number of electrons per nucleus and thus should be more efficient. Same with iron and lead: their ionization energy loss is lower than the loss in aluminum (fig 4) and thus should be less efficient.

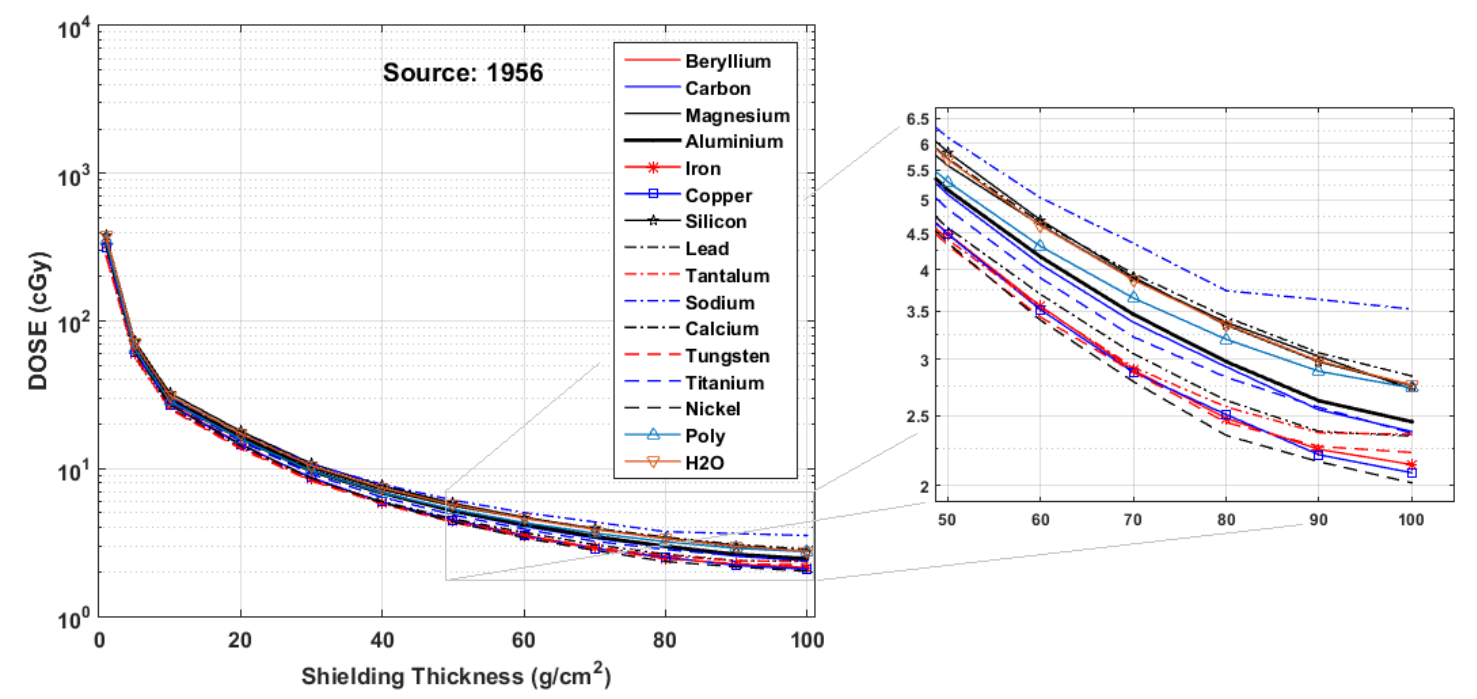

Figure 25 Dose Profiles in Different Materials (SPE source: 1956)

In fact, an opposite conclusion can be drawn by comparing figure 25 with figure 4 . It is unlikely that the reason is due to erroneous setup of the model in Fluka since aluminum was confirmed with the Rojdev paper. This contradiction is probably due to the 1956 SPE which has particularly long high energy tail. This event contain protons with energies much higher than a few tens of MeV. For such particles, the energy loss in a shield might no longer be proportional to Z/A ratio. This is a possible explanation why materials with lower ionization energy loss perform better when source is the 1956 SPE [9]. In order to test this assumption, the same plot as figure 25 was constructed for 1989 event. According to the results shown in figure 26, it can be concluded that all profiles for the 1989 SPE are lower in magnitude by about 1 cGy compared to 1956 envent. For this solar event, the material's dose profiles are in accordance with the Z/A of the material: both hydrogen and water perform better. 


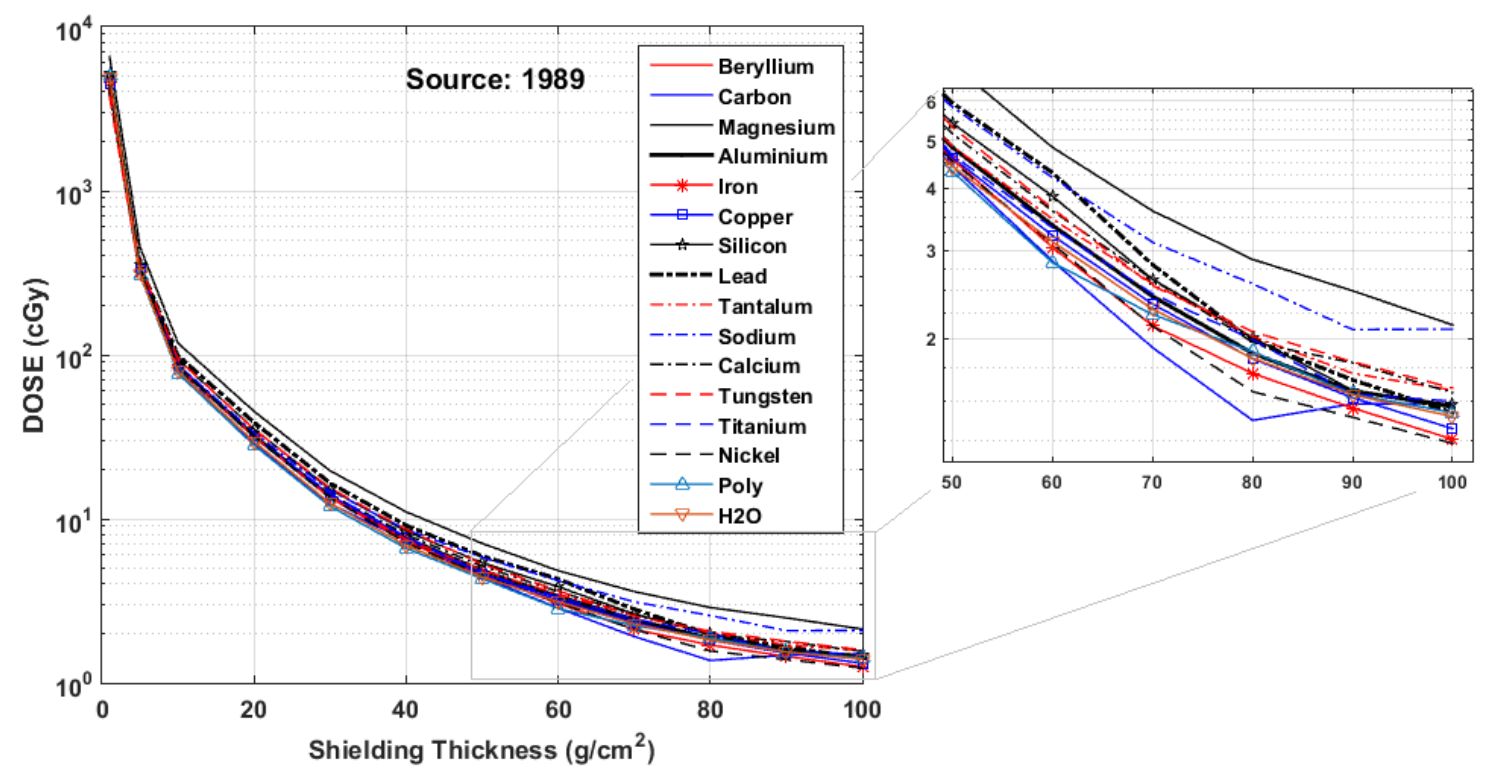

Figure 26 Dose Profiles in Different Materials (SPE source: 1989)

As for the target, it was important not only to get the total absorbed dose but also to understand what secondary particles, produced in the shield, contribute most to the total dose. Fluka allows to score dose specifically due to certain particle. If one knows what secondaries are produced in the shield, it is possible to determine how much each of these particles contribute to the total dose absorbed by the target. The run summary, found in the Fluka's output file, provide information about the secondary particles [15]. It includes percentage breakdown for the secondaries abundance. The output file shows the following three energetic particles to be most abundant for all tested materials: protons, neutrons and electrons. Therefore, the target dose is reported in terms of the dose components due to each of these three particles.

Since SPE source was modeled only as a fluence of protons, any contribution to the target dose other than from protons must be due to secondary particles such as alpha, electrons and neutrons. Figure 27 shows the fluence of the main target's dose contributors (protons, electrons, neutrons). Part $a$ is the particles fluence in between shield and target while part $b$ shows proton fluence only between first few layers of the shield. Comparing the amount of uncertainty in electron fluence with the uncertainty in neutrons for the given number of history particles, one can argue that electrons are much less abundant than neutrons yet their contribution is again higher than neutrons. The fact that even small amount of electrons (the second contributor to the total target dose) and that electrons together with protons contribute more than $90 \%$ means that ionizing radiation is what responsible for most of the dose absorbed by target. Also, the reason why proton's contribution is 
significantly larger than contribution from the rest of the particles is because protons are the only charged particles leaving shield with high energies.

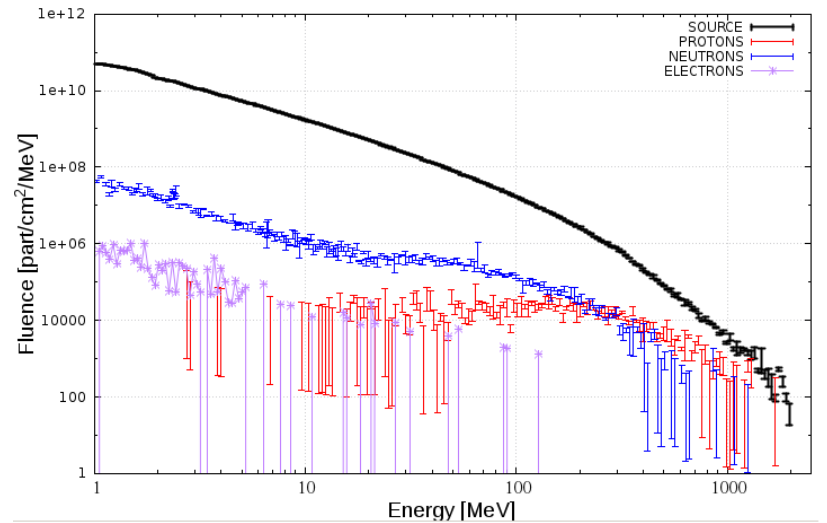

(a)

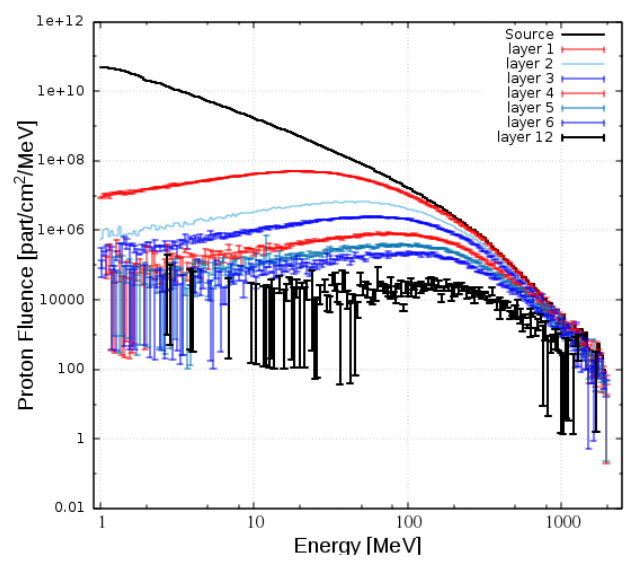

(b)

Figure 27 (a) Fluence of protons, electrons and neutrons between shield and target, (b) Proton fluence between some shield layers. The source fluence is added for comparison. (Aluminum. Source: 1989)

Part $b$ of figure 27 helps to understand what happens to the primary protons (from the source). The first layer significantly reduces the proton fluence while the efficiency of each subsequent layer to "filter out" protons gets smaller. As expected, the shield's efficiency drops with increasing in proton energy as the fluence difference of the $1 \mathrm{GeV}$ protons across the whole shield is significantly smaller than of $10 \mathrm{MeV}$ protons.

Figure 28 demonstrate the dose absorbed by target due to the 1956 and 1989 events. In order to visualize the relative dose contribution by each type of the secondary particle, each dose value (column) is broken into dose due to protons, electrons, neutrons, alphas and others. The percent contribution of each particle is nearly the same for all materials with protons being the dominant contributor (<87\% for 1956 ; < $85 \%$ for 1989 ). 


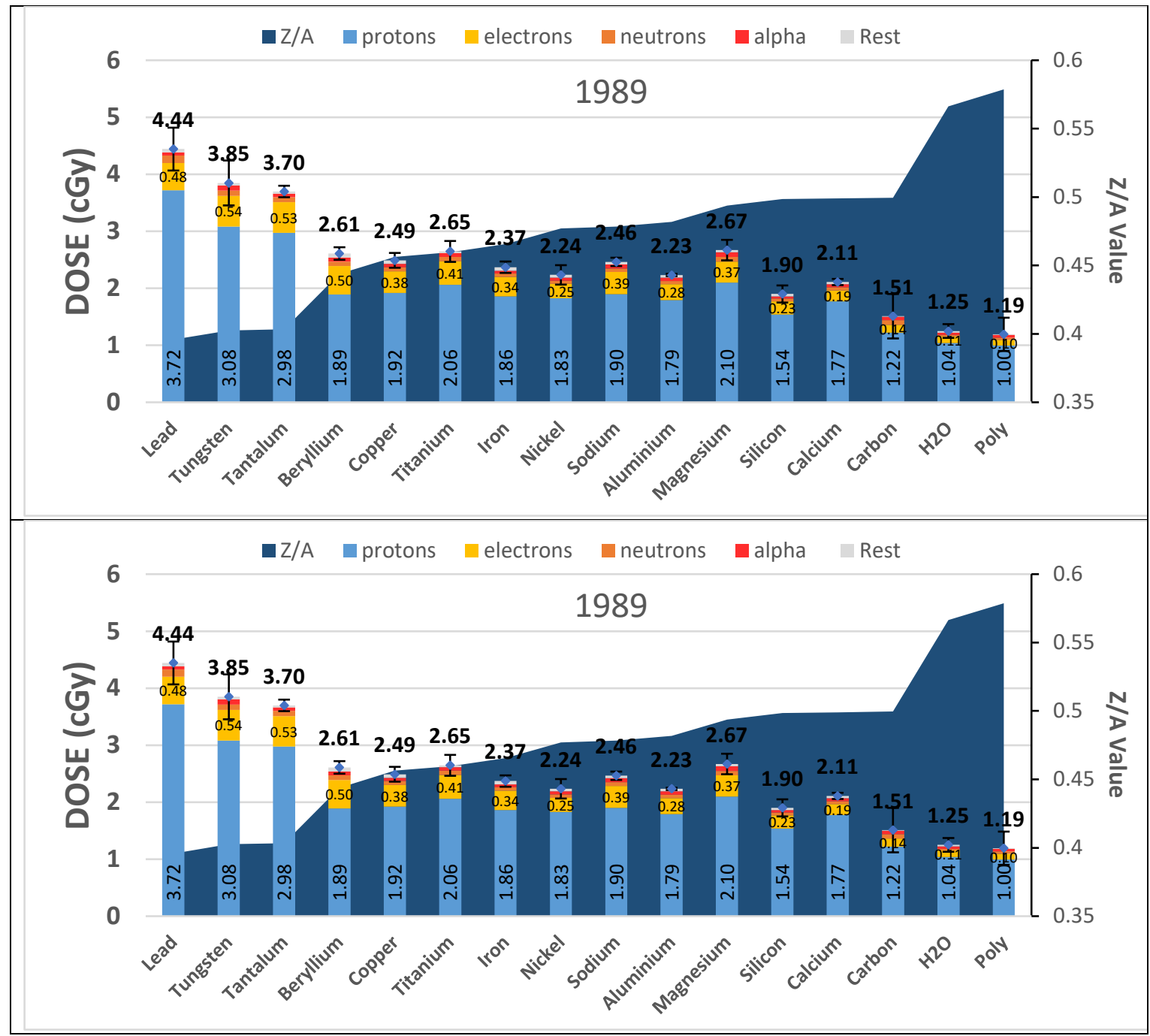

Figure 28 Dose Absorbed by Target for the 1956 and 1989 SPEs (Shield: 100g/cm2). Total doses are given on top of each bar. Values for protons and electrons are also included. The area on the background is Z/A with own axis on the right

Electrons are the next largest dose contributors but the dose associated with them is much smaller $(<14 \%$ for 1956; < $20 \%$ for 1989). Neutrons, alphas and other particles combined contribute less than $9 \%$ of the total absorbed dose. The area plot on the background of figure 28 represent the Z/A for each test material. Materials in the plot are arranged in the increasing order of their Z/A values. Effective atomic number used to calculate Z/A values for water and polyethylene was calculated as follows:

$$
Z_{\text {eff }}=\sqrt[2.94]{f_{1} \times\left(Z_{1}\right)^{2.94}+f_{2} \times\left(Z_{2}\right)^{2.94}+f_{3} \times\left(Z_{3}\right)^{2.94}+\ldots},
$$

Theoretically, as this ratio increases from left to right, the dose received by a target should get smaller because more energy is absorbed in a shield rather than in the target. Although the target doses in both plots 
seems to show no decreasing trend, the three materials (lead, tungsten and tantalum) with Z/A significantly smaller than the rest of the materials do indeed have the largest total doses and polyethylene, with the highest Z/A value, has the lowest dose.

It is important to notice here that both geometrical thickness and mass of each layer varies with material but since the purpose of this experiment is to compare performance of different materials, some variable must stay constant. Here, such variable is area density. For linear geometry such as cylinder, fixed area density means that layers of different materials have different thicknesses while their masses are equal no matter what material they are made of. This is because a mass relates to the flux area linearly, which in turn is constant in case of cylinder. However, in case of sphere, the flux area changes with radius and thus both thickness and mass changes from layer to layer.

For practical reasons, perhaps a more useful way to compare performance of different materials is be to fix the overall mass of a shield allowing its thickness to vary. Figure 29 compares two approaches: (a) - shield with fixed area density and (b) - shield with fixed mass. Both are subject to 1989 SPE. Part $a$ is the same as figure 28 but with the shield's mass superimposed. Part $b$ is the case when the mass of a shield is fixed at 100 kg. Doses are arranged from the smallest to the highest. The red background area is the reference dose limit equal to the dose due to aluminum shield. Performance of the tested materials appears to be similar to the case of fixed area density: the polyethylene shield yields the lowest target dose while the lead shield yields the highest dose. In fact, among seventeen tested materials, only four exhibit better performance than aluminum: polyethylene, carbon, water and silicon. 


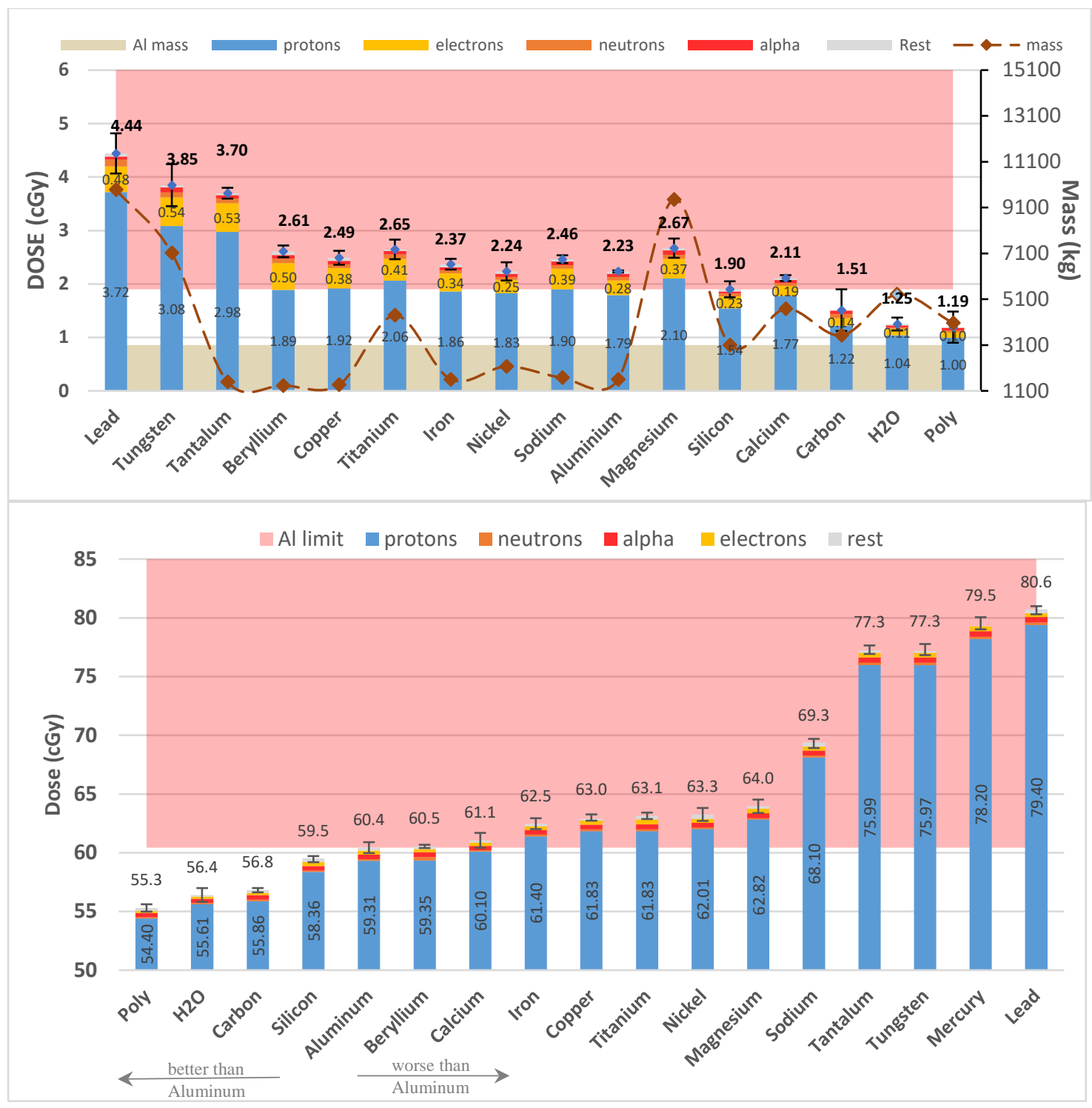

Figure 29 (a) Dose Absorbed by Target. Mass of a Shield is Added Instead of Z/A (SPE source: 1989. Shield: $100 \mathrm{~g} / \mathrm{cm}^{2}$

(b) Dose Absorbed by Target (SPE source: 1989). Fixed Shield Mass (100kg)

\subsection{Combination of Materials (SPE)}

So far, only the performance of a shield made entirely of a single material was investigated. Does combination of different materials affect the overall efficiency of a shield? To answer this question, several combinations of materials were investigated. Each combination set involve a pair of materials:

- lead - aluminum

- $\quad$ lead - polyethylene 
- $\quad$ lead - carbon

- aluminum - carbon

These materials were choosen due to their high performance found in the previous experiment. The source was chosen to be the 1989 event and the experiment was repeated for two shield thicknesses: 100 and 10 $\mathrm{g} / \mathrm{cm}^{2}$. The following combinations were used:

- $1 / 4$ of shield thicness of one material and $3 / 4$ of the other material

- Half of one material, half of the other material

- The same combinations but with materials switched places

- A thin outer layer of certain material, the rest layers are of the other material

Table 14 summarizes all combination sets used in this experiment. These combinations were chosen to test whether the structure of a typical shield of a high energy proton accelerator can be applied in a spacecraft's shielding. The accelerator shield consists of a layer of high-Z material followed by a low-Z material. The high$\mathrm{Z}$ materials reduce the proton's energy and low-Z material reduces it farther down to thermal energy [9]. In this experiment the following materials with low-Z were chosen: polyethylene, water and carbon. Lead was used as a high-Z material. Combinations of lead with aluminum was also tested because of particular interest in aluminum as it is the most prevalent material in spacecraft. The goal was to combine layers of different materials such that the overall dose absorbed by a target behind this shield would be lower than the reference dose which is due to the aluminum shield of the same areal density. The overall mass of the shield must be smaller or at least equal to the reference mass. 


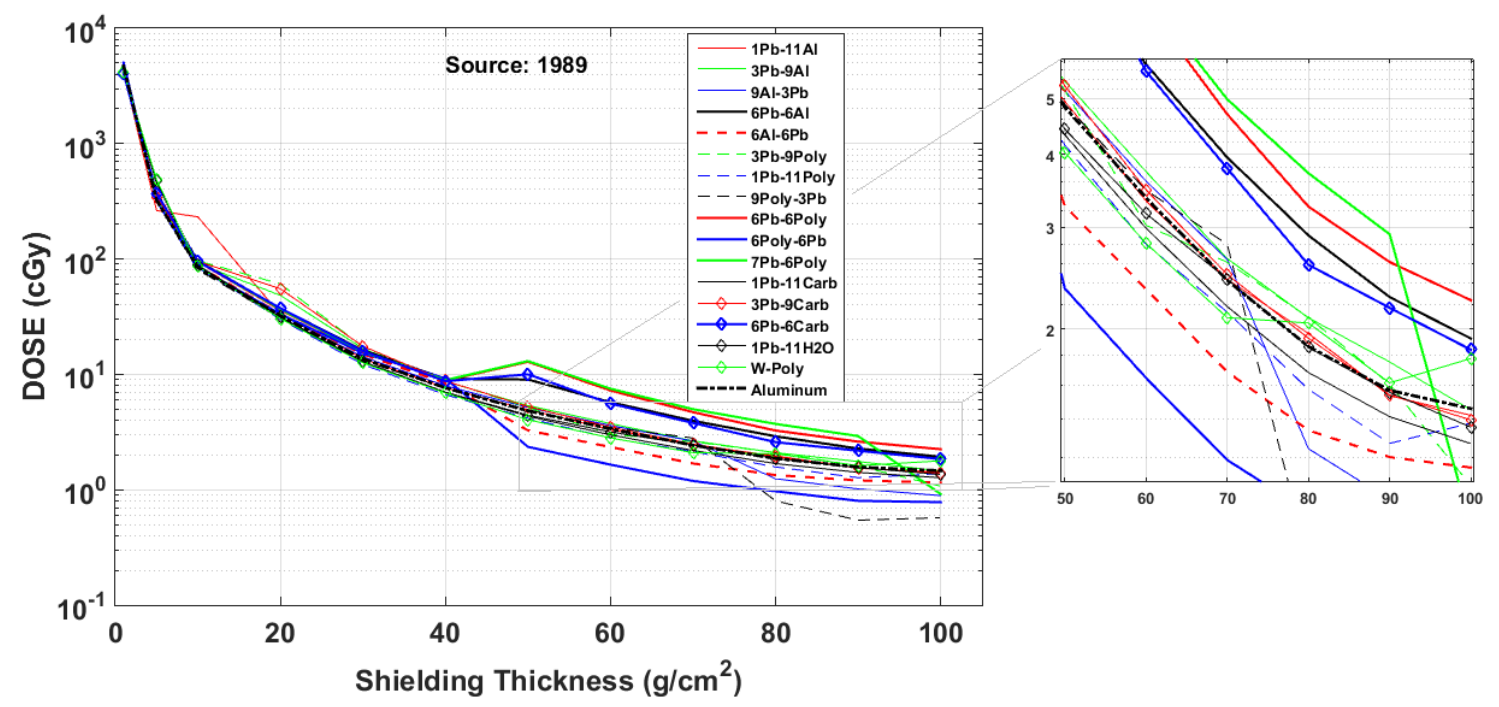

Figure 30 Dose Distribution Within a $100 \mathrm{~g} / \mathrm{cm} 2$ Shield Constructed Out of the Layers of Different Materials

Figure 30 shows the dose profiles of all tested combinations while the corresponding target's doses for a shield of 100 and $10 \mathrm{~g} / \mathrm{cm}^{2}$ thick can be found in figure 31. The material combination sets in figure 31 are arranged in the same order as in table 14 for convenience. In order to quickly assess the performance of a given combination set, two background areas were added to the figure. The red area on top of the figure sets the reference dose. Any combination having a dose that crosses this area performs worse than the aluminum shield. The area at the bottom of the figure sets the reference mass. If a certain combination does not reach the red zone but weights more than the reference, it still performs worse than aluminum. The mass of each combination is given as a blue dashed line.

The first thing to notice in figure 31 is that the shielding scheme used in a high-energy proton accelerator does indeed help to reduce the dose received by a target. The combination sets where the polyethylene layers follow the lead layers are indeed significantly lower than the reference. Also, when high and low-Z layers are switched places, the resultant dose is higher. However, all the polyethylene combinations, which yield lower doses than the reference, are made of significantly heavier shields than the reference and therefore are not beneficial. The only combinations that yield lower doses and weight about the same as the reference are $1 \mathrm{~Pb}$ $11 \mathrm{Al}$, 3Car-9Al and 6Car-6Al yet their advantage over the Aluminum shield is only up to $10 \%$. 
Table 14 Combiation of Materials

\begin{tabular}{|c|c|c|c|c|c|c|c|c|c|c|c|c|c|c|c|c|c|}
\hline \multicolumn{2}{|c|}{ layers } & \multicolumn{5}{|c|}{ Lead - Aluminum } & \multicolumn{6}{|c|}{ Lead - Polyethylene } & \multicolumn{3}{|c|}{ Lead - Carbon } & \multirow{2}{*}{$\begin{array}{c}\text { 3Car-9Al } \\
\text { Carb }\end{array}$} & \multirow{2}{*}{$\begin{array}{c}\text { 6Car-6Al } \\
\text { Carb }\end{array}$} \\
\hline \multicolumn{2}{|c|}{1 (outer) } & lead & lead & $\mathrm{Al}$ & lead & $\mathrm{Al}$ & lead & lead & Poly & lead & Poly & lead & lead & lead & lead & & \\
\hline \multirow{2}{*}{\multicolumn{2}{|c|}{$\frac{2}{3}$}} & $\mathrm{Al}$ & lead & $\mathrm{Al}$ & lead & $\mathrm{Al}$ & Poly & lead & Poly & lead & Poly & lead & Carb & lead & lead & Carb & Carb \\
\hline & & $\mathrm{Al}$ & lead & $\mathrm{Al}$ & lead & $\mathrm{Al}$ & Poly & lead & Poly & lead & Poly & lead & Carb & lead & lead & Carb & Carb \\
\hline \multicolumn{2}{|c|}{$\frac{3}{4}$} & $\mathrm{Al}$ & $\mathrm{Al}$ & $\mathrm{Al}$ & lead & $\mathrm{Al}$ & Poly & Poly & Poly & lead & Poly & lead & Carb & Carb & lead & $\mathrm{Al}$ & Carb \\
\hline \multicolumn{2}{|c|}{5} & $\mathrm{Al}$ & $\mathrm{Al}$ & $\mathrm{Al}$ & lead & $\mathrm{Al}$ & Poly & Poly & Poly & lead & Poly & lead & Carb & Carb & lead & $\mathrm{Al}$ & Carb \\
\hline \multicolumn{2}{|c|}{6} & $\mathrm{Al}$ & $\mathrm{Al}$ & $\mathrm{Al}$ & lead & $\mathrm{Al}$ & Poly & Poly & Poly & lead & Poly & lead & Carb & Carb & lead & $\mathrm{Al}$ & Carb \\
\hline \multicolumn{2}{|c|}{7} & $\mathrm{Al}$ & $\mathrm{Al}$ & $\mathrm{Al}$ & $\mathrm{Al}$ & lead & Poly & Poly & Poly & Poly & lead & Poly & Carb & Carb & Carb & $\mathrm{Al}$ & $\mathrm{Al}$ \\
\hline \multicolumn{2}{|c|}{8} & $\mathrm{Al}$ & $\mathrm{Al}$ & $\mathrm{Al}$ & $\mathrm{Al}$ & lead & Poly & Poly & Poly & Poly & lead & Poly & Carb & Carb & Carb & $\mathrm{Al}$ & $\mathrm{Al}$ \\
\hline \multicolumn{2}{|c|}{9} & $\mathrm{Al}$ & $\mathrm{Al}$ & $\mathrm{Al}$ & $\mathrm{Al}$ & lead & Poly & Poly & Poly & Poly & lead & Poly & Carb & Carb & Carb & $\mathrm{Al}$ & $\mathrm{Al}$ \\
\hline \multicolumn{2}{|c|}{10} & $\mathrm{Al}$ & $\mathrm{Al}$ & lead & $\mathrm{Al}$ & lead & Poly & Poly & lead & Poly & lead & Poly & Carb & Carb & Carb & $\mathrm{Al}$ & $\mathrm{Al}$ \\
\hline \multicolumn{2}{|c|}{11} & $\mathrm{Al}$ & $\mathrm{Al}$ & lead & $\mathrm{Al}$ & lead & Poly & Poly & lead & Poly & lead & Poly & Carb & Carb & Carb & $\mathrm{Al}$ & $\mathrm{Al}$ \\
\hline \multicolumn{2}{|c|}{12 (inner) } & $\mathrm{Al}$ & $\mathrm{Al}$ & lead & $\mathrm{Al}$ & lead & Poly & Poly & lead & Poly & lead & lead & Carb & Carb & Carb & $\mathrm{Al}$ & $\mathrm{Al}$ \\
\hline \multirow{2}{*}{ 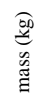 } & 100 & 3103 & 3080 & 4530 & 2771 & 1731 & 9880 & 9723 & 5072 & 7740 & 2489 & 6044 & 4063 & 4023 & 3501 & 3269 & 9880 \\
\hline & 10 & 128 & 128 & 122 & 126 & 118 & 158 & 157 & 136 & 151 & 123 & 143 & 133 & 133 & 130 & 128 & 128 \\
\hline \multirow{2}{*}{ 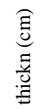 } & 100 & 64.43 & 34.23 & 28.58 & 25.75 & 20.11 & 105 & 96.63 & 77.11 & 67.35 & 47.84 & 57.60 & 49.59 & 45.88 & 33.52 & 42.23 & 105.37 \\
\hline & 10 & 3.7 & 3.4 & 2.9 & 2.6 & 2.0 & 10.5 & 9.7 & 7.7 & 6.7 & 4.8 & 5.8 & 5.0 & 4.6 & 3.4 & 3.8 & 3.7 \\
\hline
\end{tabular}




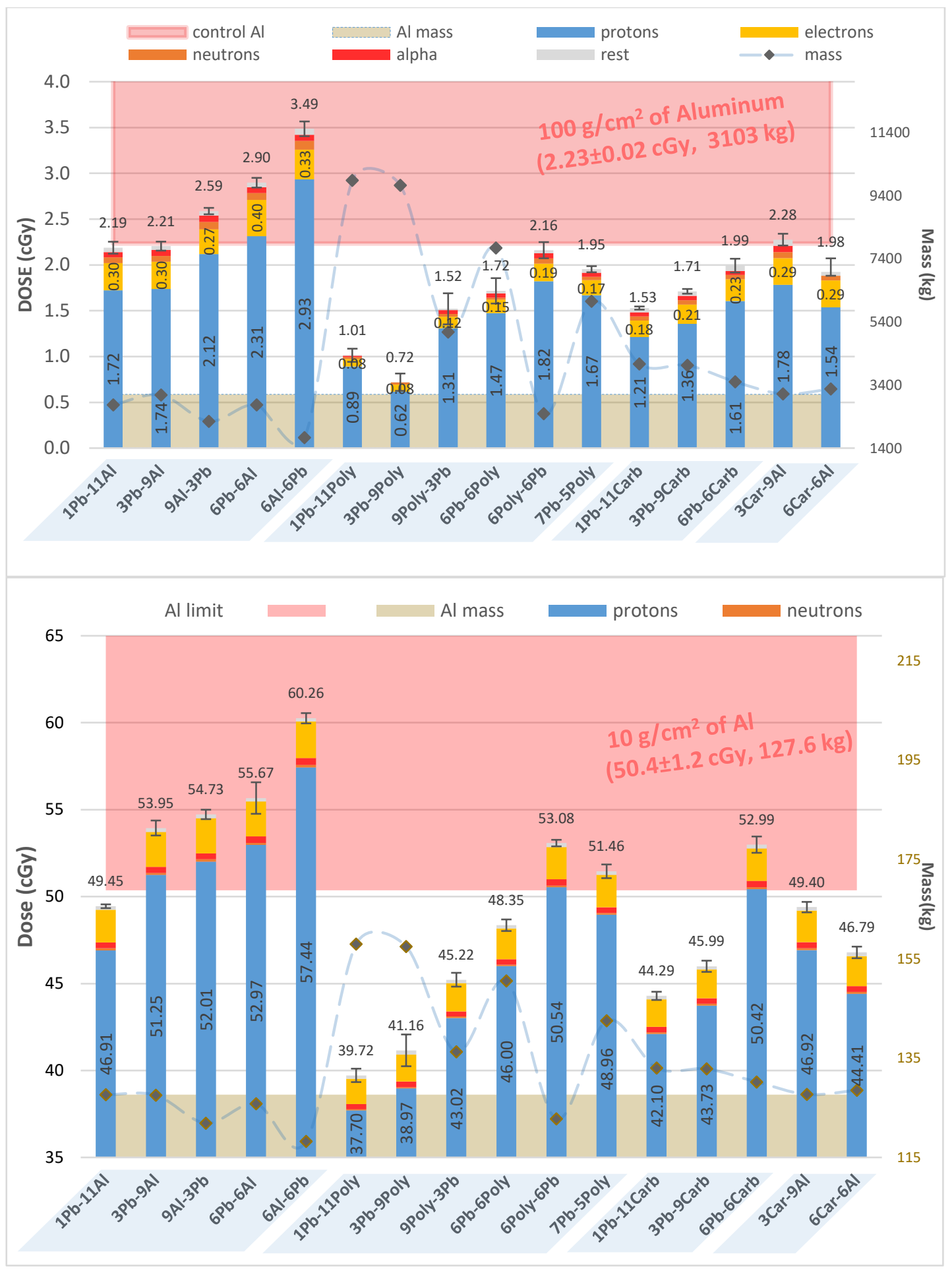

Figure 31 Target Doses Absorbed with Composite Shield of 100 and $10 \mathrm{~g} / \mathrm{cm}^{2}$ Thick. (SPE source: 1989) 


\subsection{Dose Profiles Due to GCR}

This experiment was performed to determine the effectiveness of different materials to shield from

GCR. The source is uniform and isotropic fluence that consist of six ions: protons, $\alpha$-particles, litium, Beryllium, Carbon and Boron. Figure 12 shows the energy spectrum for the first four of these ions. Using the same shielding materials as before and the simplified model (fig 24) with shield thickness of $10 \mathrm{~g} / \mathrm{cm}^{2}$. The target's absorbed doses are shown in figure 32. The first thing to notice here is the small effect of material choice; all the tested materials yield the target's dose of about $360 \mu \mathrm{Gy} / \mathrm{day}$. Including errors, the highest difference is between sodium and tantalum: $35 \mu \mathrm{Gy} /$ day. The target, shielded by sodium, absorbs a dose with a rate that is $90.7 \%$ of the rate due to tantalum shield (fig 32). In other words, the difference between the most and the least efficient materials is within about 9\%. In case of the SPE, the biggest dose difference is within $81 \%$ (fig 29a). Therefore, it can be concluded that choice of material matters more for shielding from SPEs rather than from GCR. This is due to higher energies of GCR ions.

Another thing to notice is that although protons are still dominant contributors to the target's dose, their role is estimated to be about $57 \%$ of the total dose absorbed by a target while in case of 1989 SPE this value was close to $80 \%$ (compare fig 32 with fig 31). Also, other particles such as pions, positrons and $\alpha$-particls show noticeable contribution compared to SPE case. This means that GCR creates a larger population of secondaries inside the shield and this adds more dose to the target. 


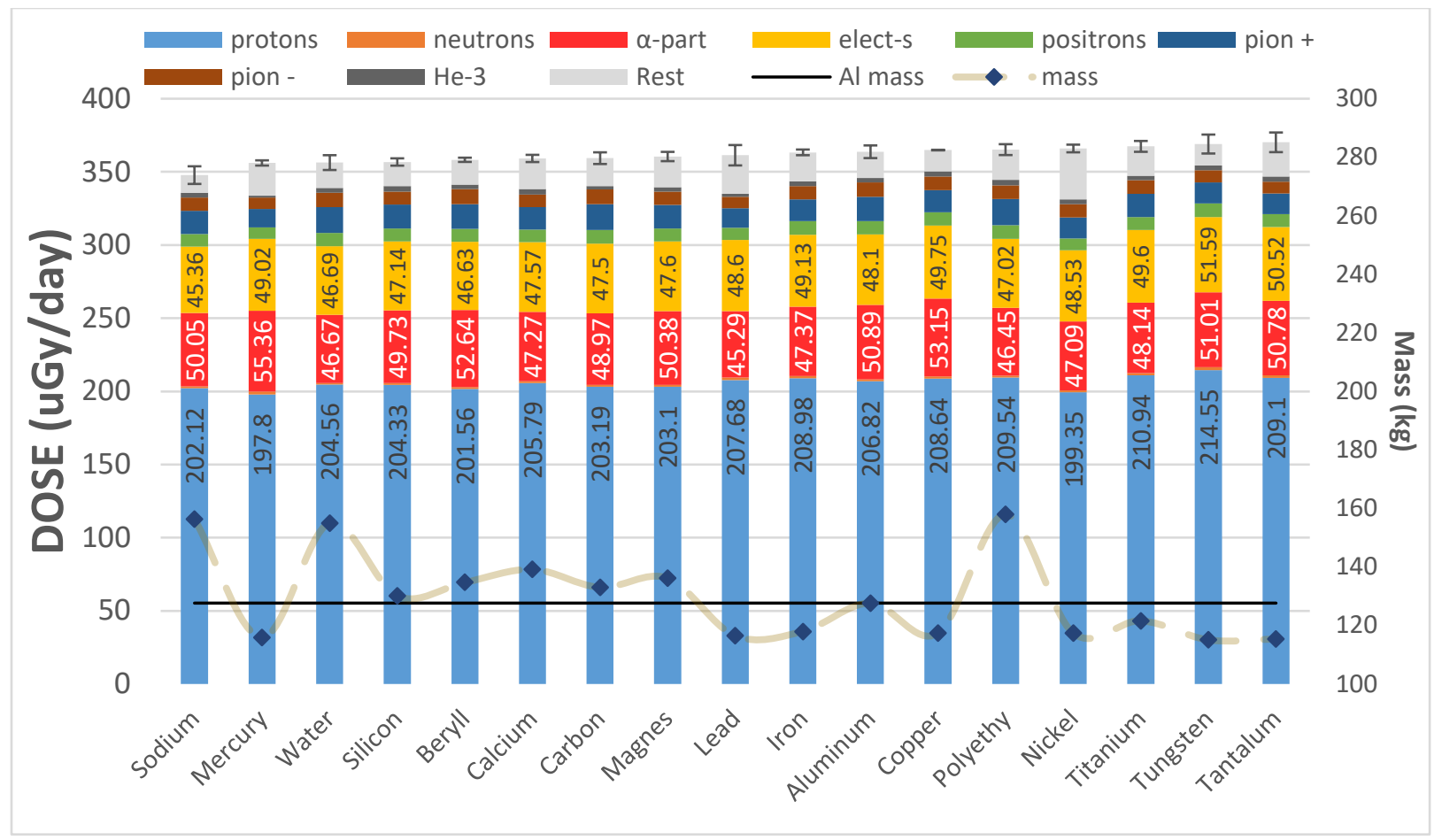

Figure 32 Dose Absorbed by Target Behind $10 \mathrm{~g} / \mathrm{cm} 2$ of a Shield Made of Single Material (GCR Source)

\subsection{Combination of materials (GCR)}

If the choice of shielding material has only a little effect on the target dose due to GCR, would a composite shield, with layers of different materials, improve the efficiency of shielding? To answer this question, the same experiment with the same combinations of materials as before was performed but this time with GCR as a source. Figure 33 shows the resultant distribution of doses arrayed in an increasing order. Again, an efficient shield is defined as the one that reduses the target's dose lower than the reference and at the same time as the one that weighs less or the same as the reference. According to the figure, if errors are included, all combinations to the left from 9Poly-3Pb result in a lower dose. However, only 3Carb-9Al, 6Pb-6Carb, 6Pb-6Al and 9Al-3Pb fully fit the efficiency criteria. Yet the most efficient among them, 3Carb-9Al is only $8 \%$ more advantageous than the aluminum shield. Notice that this combination is also efficient for shielding from the SPE source. This means that adding a layer of carbon with $1 / 3$ of the aluminum shield thickness reduces the target's dose by $8 \%$ due to GCR

The possible way to add a carbon layer might be in a form of tiles similar to the thermal protection tiles used in the Space Shuttle. Another way would be to make a blanket (made of material similar to the one used in a space suits) with pockets filled with powdered carbon. 


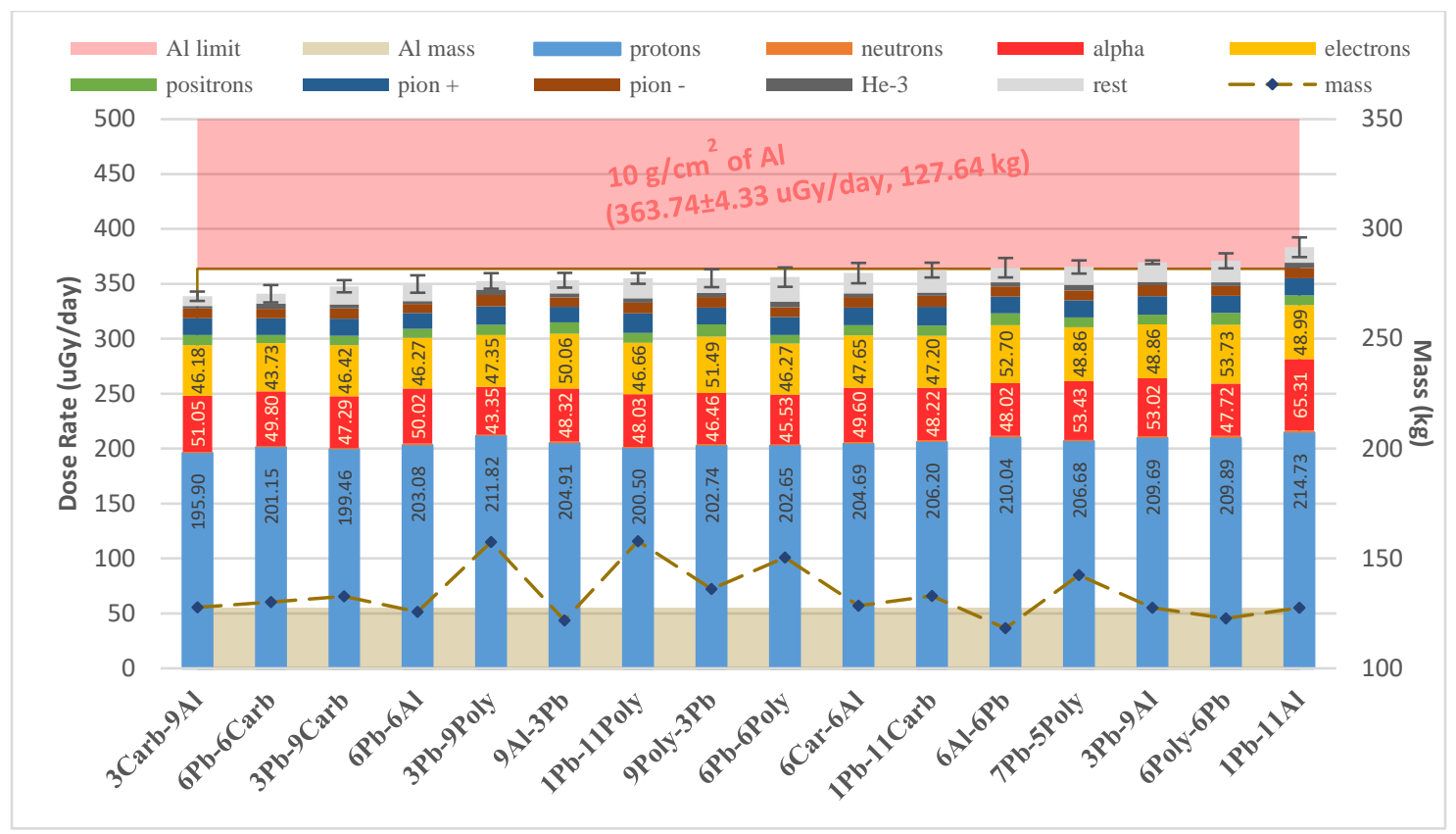

Figure 33 Target Doses Absorbed with Composite Shield of $10 \mathrm{~g} / \mathrm{cm}^{2}$ Thick. (GCR source) 


\section{CONCLUSION}

The purpose of this study was to find materials and their combination that are advantageous in terms of radiation shielding in space. This radiation is one of the major obstacles to having a human presence in deep space. Based on the data collected by the MSL spacecraft, the round trip to Mars would yield a dose that is close to a career dose limit for a 45 year old male. To evaluate the material's shielding property, the expected radiation environment was modeled. In this paper, one source of such radiation (SPE) was simulated using the Band model because this model has good agreement with observational data and allows us to model almost any SPE event happened in past sixty years.

There are different ways to evaluate the efficiency of different materials to attenuate radiation in space. Today several transport codes can simulate the necessary conditions and estimate the dose absorbed by target shielded by material of interest. This study employs the Monte Carlo code called FLUKA because of its high accuracy and availability. The advantage of FLUKA is that it models complex objects using the Constructive Solid Geometry Module and it has built-in tools to model the isotropic GCR environment. The GCR at solar minimum was chosen because that is when the GCR intensity is highest.

Before FLUKA was used for this study, its output was validated with the results reported in published papers. An intention of this study was to find a material that would outperform aluminum - the most prevalent material in spacecraft. The test model was chosen to be as realistic as possible but at the same time would not requiring too much computational time.

It was found that hydrogen rich materials do not always outperform other materials and their effectiveness

depends on the source energy spectrum. Specifically, polyethylene, even though this material has high ionization energy loss, is less advantageous than copper or iron in case of the 1956 SPE source but it is more advantageous in case of the 1989 SPE. It is assumed that the reason for this is a particularly long high energy tail of the 1956 event. It was found that in both SPE and GCR cases protons contribute the most to the overall dose absorbed by the target. Electrons are the second largest contributor. The shield of $100 \mathrm{~g} / \mathrm{cm}^{2} \mathrm{efficiently}$ filters out the SPE protons with energies up to about $100 \mathrm{MeV}$ and its efficiency drops quickly for protons with higher energies. 
In case of SPE, the materials that outperform aluminum are polyethylene, water, carbon and silicon (in decreasing order). The shielding scheme used in a high-energy proton accelerator, which is where the layer of low-Z material follows the high-Z material, does indeed helps to reduce dose received by a target. The only combinations that yield lower target doses and have about the same weight as the reference are $1 P b-11 \mathrm{Al}$, 3Car-9Al and 6Car-6Al yet their advantage over the aluminum shield is up to $10 \%$.

The performance of materials and their combinations were compared by fixing the overall area density of a shield. One test was performed with the shield's mass instead of area density being fixed. This test demonstrated the same results as with fixed area density case.

The GCR source was modeled as a fluence of six ions. It was found that the choice of material used for shielding from GCR has much smaller effect on the efficiency of a shield than the case of SPE source. The highest difference in the rate of dose absorption was found between sodium and tantalum shields with sodium being more efficient shielding material. However, the difference between them is less than $9 \%$.

Besides electrons, other secondary particles generated inside the shield for the GCR case are pions, $\alpha$ particle and others. The following composite shields made of layers of different materials 3Carb-9Al, 6Pb6Carb, $6 \mathrm{~Pb}-6 \mathrm{Al}$ and $9 \mathrm{Al}-3 \mathrm{~Pb}$ show better performance than aluminum shield. The most advantageous of them, 3Carb-9Al is only $8 \%$ more efficient. 3Carb-9Al combination is also the most efficient when the source is a SPE making this combination the first candidate for testing in space. 


\section{FUTURE WORK}

Several improvements and additional experiments can be performed in order to farther investigate the shielding properties of materials. One thing to modify is the radiation source. The two types of sources (GCR and SPE) in this study were modeled separately meaning either one of them were included in each experiment. A more realistic model would be the one where both sources are present at the same time. The galactic radiation is constantly being modulated by solar activity. The Forbush Decrease is an extreme manifestation of such an interaction. The best practice would be to generate the FLUKA's source according to the modulation equation which calculates the fluence of energetic particles originating from both sources at the same time.

This study focused only on shielding materials that are made of a number of elements from periodic table, which include mostly metals with homogeneous structure. Therefore, the next step in this study could be to test materials with porous structure filled with different gases such as air or gases with high Z/A ratio such as hydrogen or helium. Such porous structures could be made of a high-Z matrix which would cause energetic particles traversing through them to disintegrate into secondary particles which in turn would be attenuated inside gas pockets. The shape of the pockets and proportion of solid to gas in such porous structures is also worth to evaluating.

Another thing worth investigating is whether gas pressure and temperature has an effect on radiation attenuation. It would be interesting to find the relationship between these two parameters as well as the attenuation properties of different gases. Although liquid hydrogen was not tested in this study, it is being reported in many papers as the best shielding material. To be in liquid state, however, hydrogen has to be kept under a very specific temperature and above a specific pressure which are hard to maintain in space. If experiments show that the attenuation characteristics of hydrogen don't rapidly degrades with changing state from liquid to gas, it would be much easier to implement hydrogen in radiation shielding. The aforementioned scheme with porous materials may be one such implementation. Therefore, it would be helpful to have a 3D diagram with a gas phase diagram (temperature vs pressure) on the bottom and an attenuation parameter of that gas on the third axis. This can be simulated by performing a series of test runs using FLUKA where these two parameters of a hydrogen would be changed for each test run. 


\section{BIBLIOGRAPHY}

1. Adams, J, and et al. 2005. "Revolutionary Concepts of Radiation Shielding for Human Exploration of Space."

2. Aghara, S. 2015. "Shielding evaluation for solar particle events using MCNPX, PHITS and OLTARIS codes." Life Sciences in Space Research 4: 79-91. doi:10.1016/j.lssr.2014.12.003.

3. Atwell, W, and et al. 2011. "Spectral Analyses and Radiation Exposures from Several." 41st International Conference on Environmental Systems. doi:doi:10.2514/6.2011-5253.

4. Ballarini, F, and et al. 2006. "Modelling human exposure to space radiation with different shielding: The fluka code coupled with anthropomorphic phantoms." Journal of Physics: Conference Series 41: 135-142. doi:10.1088/1742-6596/41/1/012.

5. Belov, A, and et al. 2014. "Coronal Mass Ejections and Non-recurrent Forbush Decreases." Solar Physics 289 (10): 3949-3960.

6. Cucinotta, F A, M-H Y Kim, and L J Chappell. 2012. "Evaluating Shielding Approaches to Reduce Space Radiation Cancer Risks." NASA TM-2012-217361. https://three.jsc.nasa.gov/articles/CucinottaKimChappell0512.pdf.

7. Cucinotta, F. 2010. "Radiation Risk Acceptability And Limitations."

8. Fasso, Alberto. 2014. Personal Communication

9. Fasso, Alberto. 2012. Personal Communications http://www.fluka.org/web_archive/earchive/newfluka-discuss/4639.html.

10. Ferrari, A, and et al. 2005. FLUKA: A Multi-Particle Transport Code. CERN. doi:10.2172/877507.

11. Infantino, Angelo. 2015. Personal Communications http://www.fluka.org/web_archive/earchive/new-fluka-discuss/8621.html.

12. Jursa, A. 1985. "GALACTIC COSMIC RADIATION AND SOLAR ENERGETIC PARTICLE." In Handbook of geophysics and the space environment, 6.1- 6.3. Air Force Geophysics Laboratory, Air Force Systems Command, United States Air Force.

13. Kim, Myung-Hee Y. 2012. "Scientist of the Month." http://www.dsls.usra.edu/science/posters/. December 3-7. http://www.dsls.usra.edu/science/posters/Kim02_2013.pdf.

14. Olive , K.L, and et al. 2014. "COSMIC RAYS." In REVIEW OF PARTICLE PHYSICS, 378-379. Chinese Physics.

15. Polkovnikov, Mikhail. n.d. Personal Communication http://www.fluka.org/web_archive/earchive/new-fluka-discuss/9461.html.

16. Porta, A.; Agosteo, S. 2008. "DOUBLE-DIFFERENTIAL SPECTRA OF SECONDARY PARTICLES."

17. Rojdev, K, and W Atwell. 2015. "Hydrogen-and Methane-Loaded Shielding Materials for Mitigation." July.

18. Sala, Paola. n.d. "Cosmic Rays. Advanced FLUKA Course." https:/www.fluka.org/free_download/course/triumf2012/Lectures/AdvancedCosmicRays2012.pdf 
19. Sala, Paola. 2016. Personal Communication http://www.fluka.org/web_archive/earchive/newfluka-discuss/8810.html.

20. Sala, Paola. 2015 - 2016. Personal Communication

21. Theis, C. n.d. "Basic steps with SimpleGeo - CERN." http://fluka-course.web.cern.ch/flukacourse/Lectures_pdf/c19_1_SG-Intro.pdf.

22. Tylka, A, W Dietrich, and W. Atwell. 2010. "Assessing the Space-Radiation Hazard in Ground-."

23. Vollaire, Joachim. 2016. Personal Communication

24. Wilson, W J, and et al. 1997. "Workshop on Shielding Strategies for Human Space Exploration." NASA Workshop. Houston, TX: NASA, Langley Research Center.

25. Xapsos, M, and et al. 2000. "Characterizing Solar Proton Energy Spectra for Radiation Effects Applications." IEEE Transactions on Nuclear Science 47 (6): 2218-2223. doi:10.1109/23.903756.

26. Zeitlin, C, and et al. 2013. "Measurements of energetic particle radiation in transit to mars on the mars science laboratory." SCIENCE 340 (6136): 1080-1084. doi:10.1126/science.1235989. 